\title{
Nonmyocyte ERK1/2 signaling contributes to load-induced cardiomyopathy in Marfan mice
}

\author{
Rosanne Rouf, ${ }^{1}$ Elena Gallo MacFarlane, ${ }^{2}$ Eiki Takimoto, ${ }^{1}$ Rahul Chaudhary, ${ }^{1}$ Varun Nagpal, ${ }^{2}$ \\ Peter P. Rainer, ${ }^{1}$ Jay G. Bindman, ${ }^{2}$ Elizabeth E. Gerber, ${ }^{2}$ Djahida Bedja, ${ }^{1}$ Christopher Schiefer, ${ }^{1}$ \\ Karen L. Miller, ${ }^{1}$ Guangshuo Zhu, ${ }^{1}$ Loretha Myers, ${ }^{2}$ Nuria Amat-Alarcon, ${ }^{1}$ Dong I. Lee, ${ }^{1}$ \\ Norimichi Koitabashi, ${ }^{1}$ Daniel P. Judge, ${ }^{1}$ David A. Kass, ${ }^{1}$ and Harry C. Dietz ${ }^{2,3}$ \\ 'Division of Cardiology, Department of Medicine, and 'McKusick-Nathans Institute of Cenetic Medicine, Johns Hopkins \\ University School of Medicine, Baltimore, Maryland, USA. ${ }^{3}$ Howard Hughes Medical Institute, Bethesda, Maryland, USA.
}

\begin{abstract}
Among children with the most severe presentation of Marfan syndrome (MFS), an inherited disorder of connective tissue caused by a deficiency of extracellular fibrillin-1, heart failure is the leading cause of death. Here, we show that, while MFS mice (Fbn $7^{10396 /+}$ mice) typically have normal cardiac function, pressure overload (PO) induces an acute and severe dilated cardiomyopathy in association with fibrosis and myocyte enlargement. Failing MFS hearts show high expression of TGF- $\beta$ ligands, with increased TGF- $\beta$ signaling in both nonmyocytes and myocytes; pathologic ERK activation is restricted to the nonmyocyte compartment. Informatively, TGF- $\beta$, angiotensin II type 1 receptor (AT1R), or ERK antagonism (with neutralizing antibody, losartan, or MEK inhibitor, respectively) prevents load-induced cardiac decompensation in MFS mice, despite persistent PO. In situ analyses revealed an unanticipated axis of activation in nonmyocytes, with AT1R-dependent ERK activation driving TGF- $\beta$ ligand expression that culminates in both autocrine and paracrine overdrive of TGF- $\beta$ signaling. The full compensation seen in wild-type mice exposed to mild PO correlates with enhanced deposition of extracellular fibrillin-1. Taken together, these data suggest that fibrillin-1 contributes to cardiac reserve in the face of hemodynamic stress, critically implicate nonmyocytes in disease pathogenesis, and validate ERK as a therapeutic target in MFS-related cardiac decompensation.
\end{abstract}

Authorship note: E.C. MacFarlane and E. Takimoto contributed equally to this work.

Conflict of interest: The authors have declared that no conflict of interest exists.

Submitted: November 3, 2016

Accepted: June 29, 2017

Published: August 3, 2017

Reference information: JCI Insight. 2017;2(15):e91588. https://doi.org/10.1172/jici. insight.91588.

\section{Introduction}

Heterozygous mutations in the gene encoding fibrillin-1 (FBN1) cause Marfan syndrome (MFS), a systemic connective tissue disorder characterized by manifestations in the ocular, skeletal, and cardiovascular systems, including aortic root aneurysm (1). Heart failure is the leading cause of death in young children with MFS $(2,3)$. While the prevailing view is that cardiac decompensation is a consequence of hemodynamic load imposed by associated mitral valvular regurgitation, some clinical studies of MFS patients have reported ventricular enlargement or cardiac dysfunction out of proportion to the severity of valvular disease (4-7), suggesting either a concomitant primary dilated cardiomyopathy (DCM) or myocardial vulnerability to mechanical stress (8). Elucidation of the mechanism of cardiac dysfunction in MFS has important implications for the determination of surgical thresholds for valve repair and for development of novel medical therapies for heart failure.

Fibrillin-1-rich microfibrils are a critical component of the extracellular matrix (ECM) in many tissues (9). The Fbn $1^{C 1039 G /+}$ mouse model of MFS harbors a heterozygous cysteine substitution in an epidermal growth factor-like domain in fibrillin-1 representative of the most common class of mutation in MFS patients (1). Such mutations result in normal mRNA expression but decreased fibrillin-1 deposition in the ECM (1). Studies in Fbn $1^{\mathrm{ClO} 039 \mathrm{G} /+}$ mice have suggested that failed matrix sequestration of latent TGF- $\beta$, with consequent increased cytokine activity, drives many manifestations of MFS, including emphysema, skeletal muscle myopathy, aortic aneurysm, and myxomatous valvular disease (10-13). All of these manifestations are attenuated in $F b n 1^{\mathrm{ClO39G/+}}$ mice by pharmacological antagonism of the canonical TGF- $\beta$ signaling (Smad2/3) pathway with either TGF- $\beta$-neutralizing antibody (NAb) (10-14) or the angiotensin 
II type 1 receptor (AT1R) blocker (ARB), which suppresses expression of TGF- $\beta$ receptors and ligands $(12,14)$. AT1R-dependent signaling cascades also converge on the ERK1/2 pathway (15), which has also been shown to be activated in MFS aortas; selective inhibition of ERK1/2 activation using RDEA119 (refametinib) rescues aortic growth and wall architecture in MFS mice (16). Intriguingly, tissue analysis in non-MFS rodent and human heart failure reveals evidence for increased TGF- $\beta$ signaling (17-20) as well as increased deposition of collagens type I and III $(21,22)$, which are encoded by TGF- $\beta$-responsive genes. Furthermore, in myocardial cells, TGF- $\beta$ expression and secretion can be induced by angiotensin II (AngII) signaling (23) and mechanical stretch (24), respectively, and have been shown to drive the transcriptional changes that accompany the transition from compensated load-induced hypertrophy to heart failure (25-27).

In the myocardium, fibrillin-1 is a predominantly nonmyocyte-derived ECM protein $(28,29)$ that is localized in perivascular and perimysial spaces, closely paralleling the spatial arrangement of collagen $(20,30)$. Recent studies in $F b n 1^{C 1039 G /+}$ mice have shown late-onset development of DCM, which uniformly associates with valvular regurgitation and increased myocardial ERK1/2 signaling (31); however, these studies do not segregate the effects of perturbed hemodynamic load from intrinsic defects in the myocardium. Similarly, Fbn $1^{m g R / m g R}$ mice, in which a homozygous hypomorphic allele of $F b n 1$ causes severe deficiency of fibrillin-1 throughout development, develop DCM associated with severe valvular regurgitation (32). Conditional deletion of Fbn1, using homozygous Fbn $1^{\text {lox/lox }}$ mice in combination with a cardiomyocyte-specific $\alpha \mathrm{MHC}$-Cre-recombinase driver $\left(\mathrm{Fbn} 1^{\mathrm{\alpha MHC-/}}\right.$ ), also associates with structural and functional changes consistent with DCM (32). Although this latter study seems to point to an intrinsic defect in cardiomyocytes as the primary driver of DCM in the context of profound deficiency of fibrillin-1 present throughout development, the relevance of these findings to the broad clinical spectrum of MFS patients with heterozygous mutations that impose a relative and postnatally progressive deficiency of extracellular fibrillin-1 remains unclear.

To address these issues, we studied young $\mathrm{Fbn}^{\mathrm{C1039G/+}}$ mice (prior to valvular or myocardial dysfunction) in combination with a conditional provocation (transverse aortic constriction [TAC]) to impose acute hemodynamic load. Comprehensive assessment of cardiac function, gross morphology, and cellular signaling events in tight temporal sequence with TAC allowed a precise definition of gene-by-environment interactions in the pathogenesis of MFS-associated DCM.

\section{Results}

Fbn $1^{C 1039 G /+}$ mice are predisposed to load-induced heart failure. We performed serial echocardiography and pressure-volume (PV) loop analysis in $\mathrm{Fbn}^{\mathrm{C1039G/+}}$ mice. Echocardiography of $\mathrm{Fbn} \mathrm{1}^{\mathrm{ClO} O 3 \mathrm{G} / \mathrm{+}}$ mice showed that the presence of valvular regurgitation (mitral and/or aortic) associated directly with increased end-diastolic $(\mathrm{r}=0.82, P<0.001)$ and end-systolic $(\mathrm{r}=0.84, P<0.0005)$ diameters and inversely with fractional shortening $(\mathrm{r}=-0.7792, P<0.005)$ (Supplemental Figure 1A; supplemental material available online with this article; https://doi.org/10.1172/jci.insight.91588DS1). However, in mice that did not manifest valvular regurgitation, as assessed by color flow Doppler, cardiac dimensions and function were indistinguishable between WT and Fbn $1^{\mathrm{Clo3g} /+}$ mice (Supplemental Figure 1A). PV loop analysis, which isolates assessment of cardiac function from loading conditions on the ventricle (33), also showed that systolic and diastolic parameters were identical between WT and $\mathrm{Fbn}^{\mathrm{C1039G/+}}$ hearts in the absence of valvular regurgitation at 4 or 12 months of age (Supplemental Figure 1, B and C). Finally, up to 12 months of age, heart weights (Supplemental Figure 1D) and left ventricular activation of Smad2 (phosphorylated [pSmad2]) or ERK1/2 (phosphorylated [pERK1/2]) was unaltered in $\mathrm{Fbn}^{\mathrm{Cl039G/+}}$ mice (Supplemental Figure 2, A-C), as were expression levels of prototypical TGF- $\beta$ target genes Ctgf and Serpine1 (Supplemental Figure 2D). Importantly, these findings suggest that, in the unstressed heart, cardiac structure and function is normal in the Fbn $1^{\mathrm{C} 1039 \mathrm{G} / \mathrm{+}}$ mouse model of MFS.

To study whether the cardiac response to increased hemodynamic load is altered in $F b n 1^{\mathrm{Cl039G/+}}$ hearts, we exposed WT and littermate $F b n 1^{C 1039 G /+}$ mice without valvular regurgitation to TAC (referred to as WT:TAC and $\mathrm{Fbn}^{\mathrm{ClO} 103 \mathrm{G} /+}$ :TAC mice), using a magnitude of aortic constriction titrated to cause a mild pressure overload (PO). Baseline cardiac dimensions and function were indistinguishable between $F b n 1^{\mathrm{Clo3g} /+}$ and WT control mice (Supplemental Figure 3A). However, after 1 week of TAC, Fbn $1^{C 1039 G /+}$ mice uniquely showed marked ventricular dilatation and dysfunction, which progressed in the ensuing 3 weeks (Figure 1, A and B). Rare $\mathrm{Fbnl}^{\mathrm{ClO39G/+}}$ and WT mice that had developed valvular regurgitation were excluded from all analyses. 
A

B
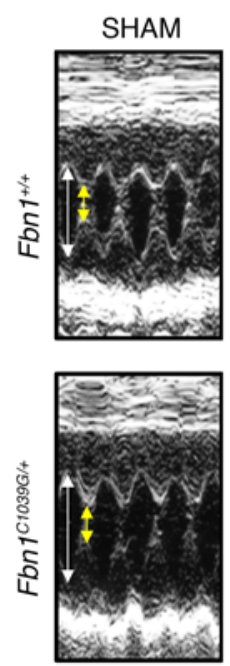
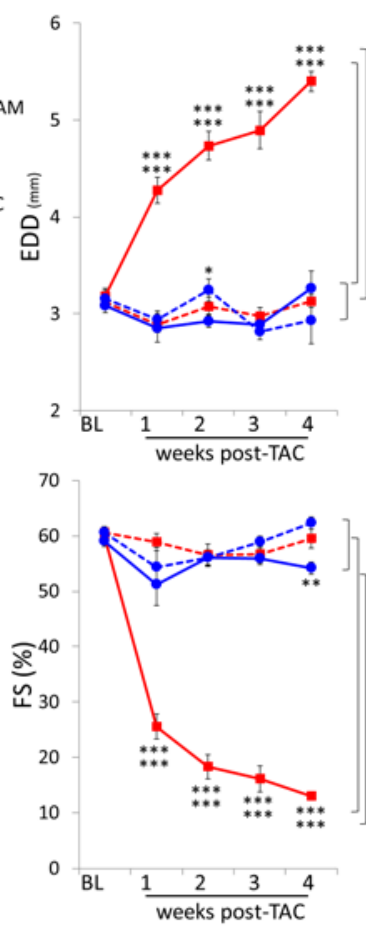
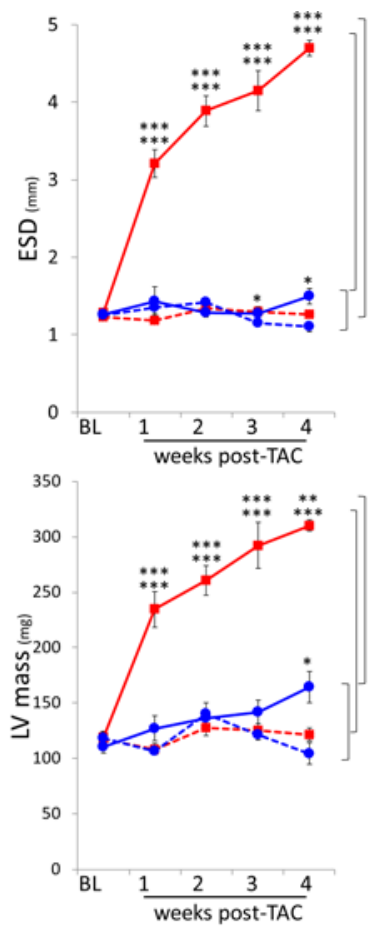

D
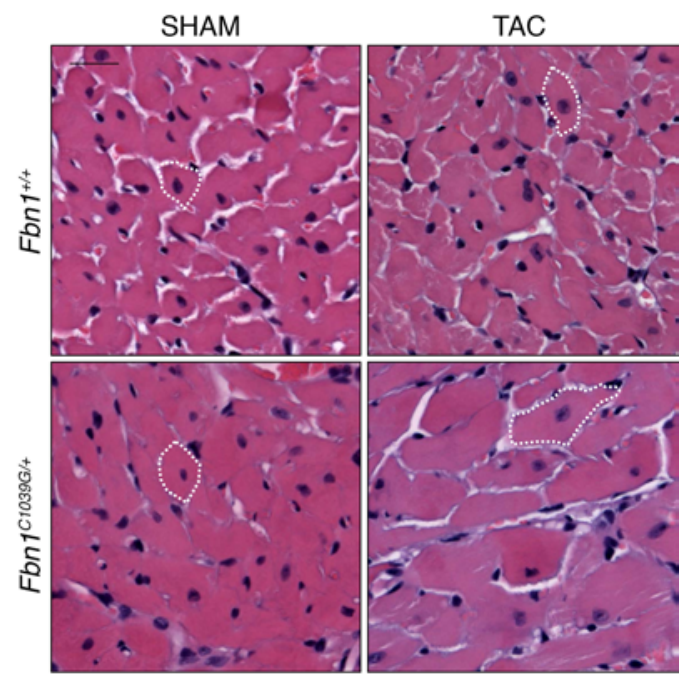

C

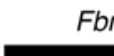

Fbn1 ${ }^{+/+}$
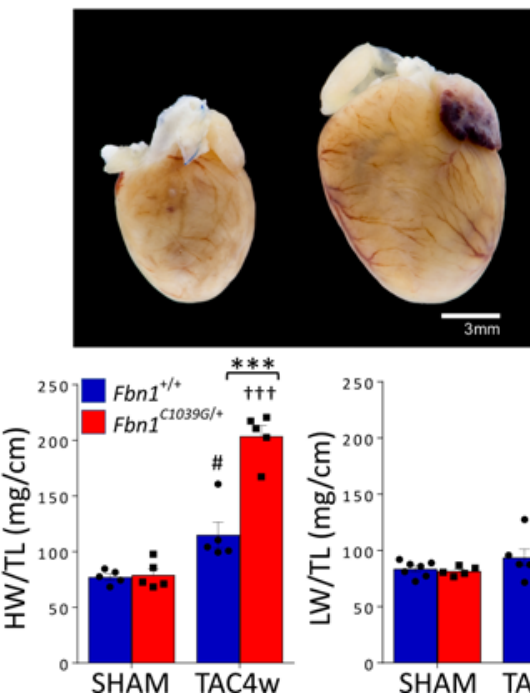

TAC
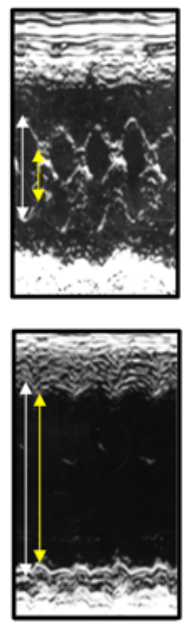

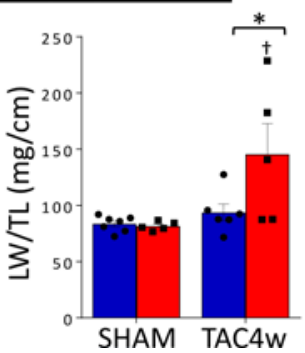

$\mathbf{E}$

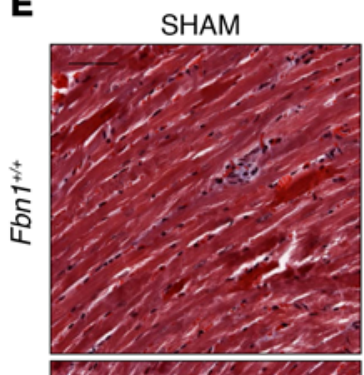

TAC
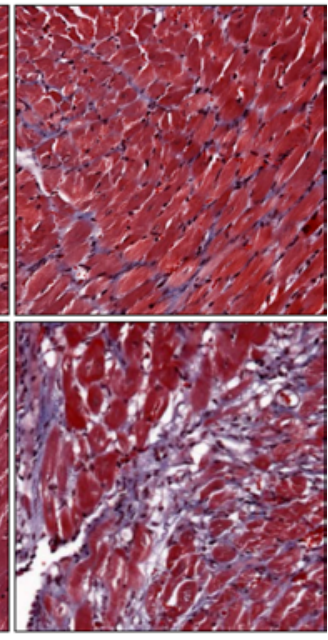

Figure 1. Fbn1 $1^{c 10396 /+}$ mice develop marked heart failure acutely from pressure overload. (A) Weekly temporal changes in cardiac dimensions and function after TAC. EDD, end-diastolic diameter; ESD, end-systolic diameter; FS, fractional shortening; LV mass, left ventricular mass; BL, baseline. Comparison pairs shown in brackets on right side of panels. $n=4-8$ per group. (B) Representative M-mode echocardiogram 4 weeks after TAC. White arrow, end-diastolic diameter; yellow arrow, end-systolic diameter. (C) Representative whole hearts and summary results for heart weight normalized to tibia length $\left(\mathrm{HW} / \mathrm{TL}\right.$ ) in $\mathrm{Fbn}^{+/+}$and $\mathrm{Fbn} 7^{11039 \mathrm{C} /+}$ mice subjected to 4 weeks of TAC. Wet lung weight (LW) was normalized to TL. $n \geq 5$ per group. ${ }^{*} P<0.05$, ${ }^{* *} P<0.01$

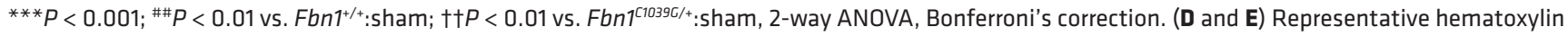
and eosin and Masson's trichrome staining of formalin-fixed heart sections from Fbn $7^{+/+}$and Fbn $7^{10396 /+}$ mice subjected to 4 weeks of TAC. Scale bars: 75 $\mu \mathrm{m}$ (D); $150 \mu \mathrm{m}$ (E). White dotted line (in D) outlines myocyte circumference. Blue stain (in E) indicates fibrosis.

Postmortem analysis after 4 weeks of TAC revealed a profound increase in heart size and mass in Fbn $1^{C 1039 G /+}$ mice compared with WT mice subjected to equivalent TAC (Figure 1C). Marked lung congestion (wet lung weight) (Figure 1C) occurred as an expected correlate with left heart failure in $F b n 1^{C 1039 G /+}$ :TAC mice. Moreover, histological analysis disclosed a marked increase in cardiomyocyte cross-sectional area and interstitial fibrosis in Fbn $1^{C 1039 G /+}$ :TAC hearts when compared with WT animals subjected to TAC (Figure 1, D and E, and Supplemental Figure 3, B and C). The expression of myocyte-specific hypertrophy-related genes, atrial 
A

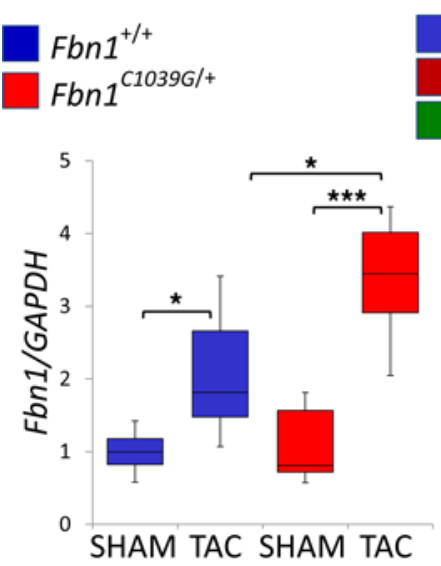

B

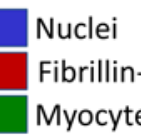

Myocyte

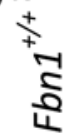

D

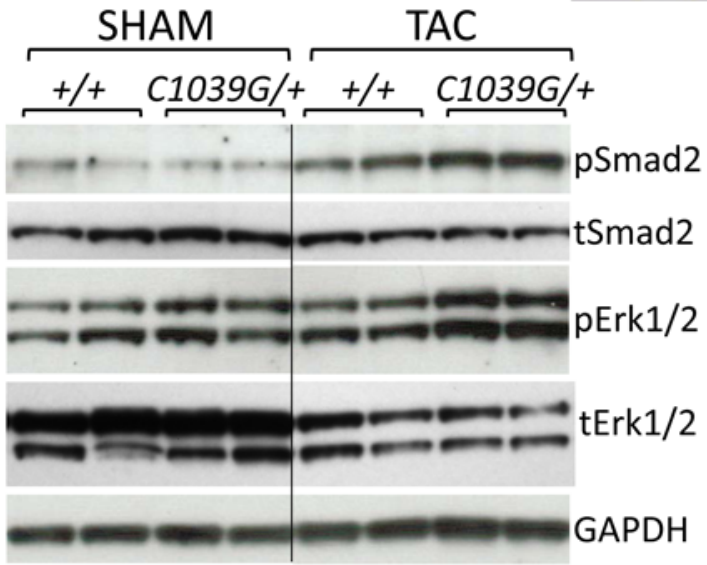

SHAM

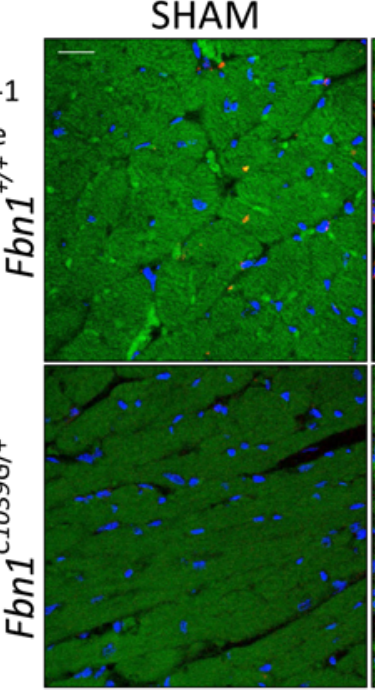

TAC

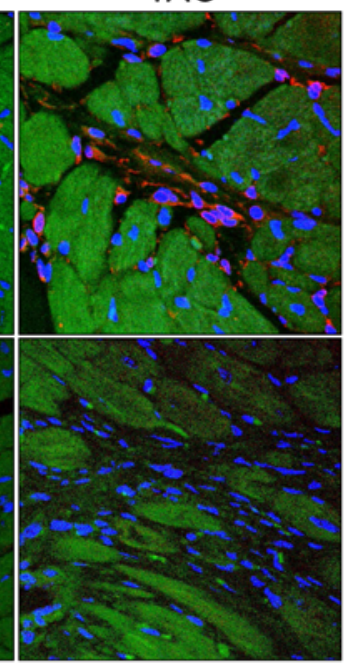

C
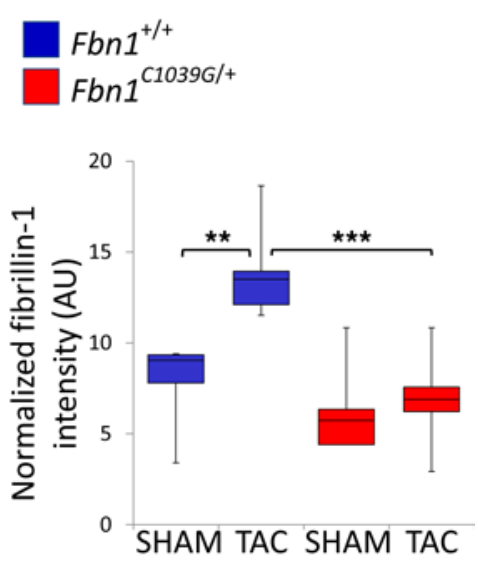

E
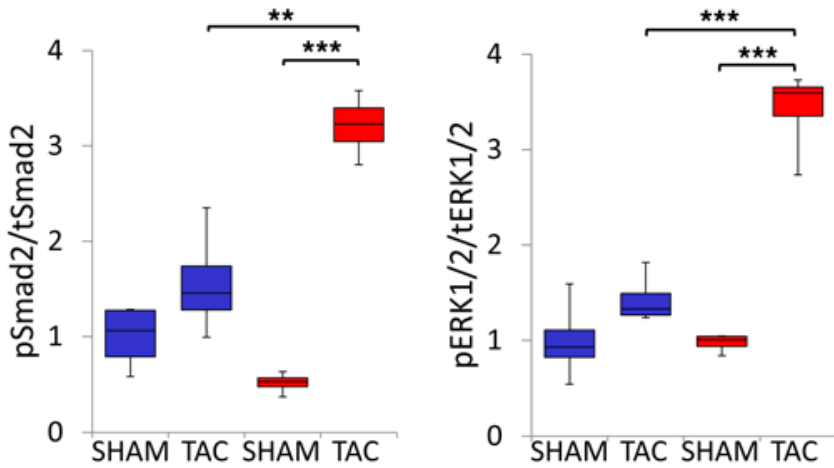

$\mathbf{F}$
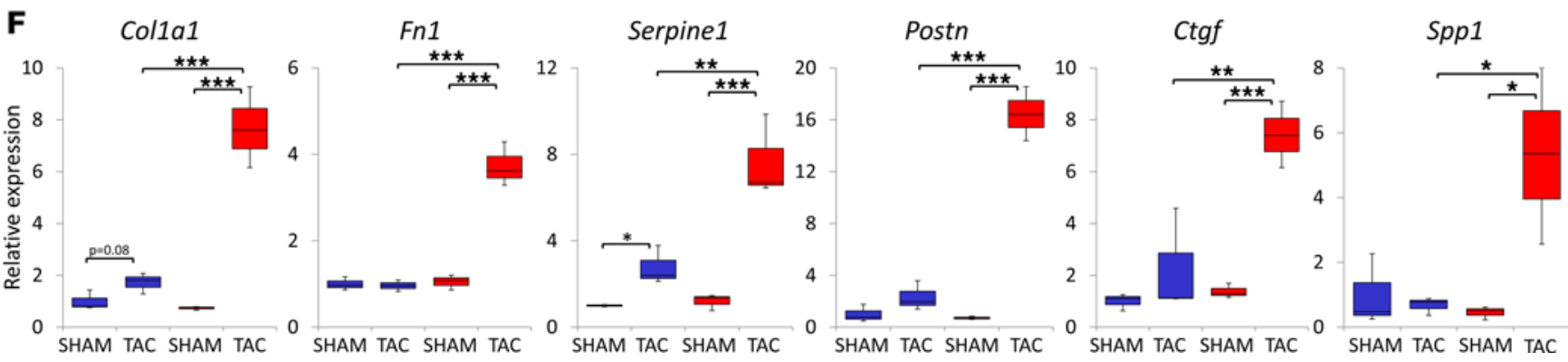

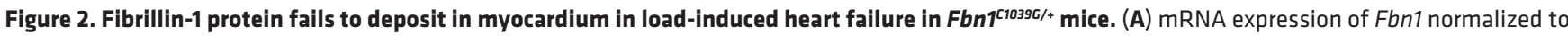
Gapdh and then to Fbn ${ }^{++}$: sham data, assessed by real-time RT-PCR. $n \geq 4$ per group. (B) Representative images of immunofluorescent fibrillin-1 staining

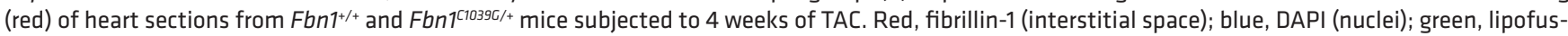
cin (myocytes). Scale bar: $20 \mu \mathrm{m}$. (C) Quantitative representation of fibrillin-1 normalized to unit area. $n=4-7$ per group. (D and E) Representative Western blot and summary quantification for phosphorylated/total ( $p / t) S \operatorname{mad} 2$ and ERK1/2 using left ventricular tissue lysates. Lanes were run on the same gel but were noncontiguous. $n \geq 4$ per group. (F) mRNA expression of TGF- $\beta$ targets, normalized to $18 \mathrm{~S}$ and then to Fbn1/++ data, assessed by real-time RT-PCR from left ventricular tissue. Col1a1, collagen type 1 a1; Fn1, fibronectin; Serpine1, plasminogen activator inhibitor, type 1; Postn, periostin; Ctgf, connective tissue growth factor; Spp1, osteopontin. $n=3-6$ per group. ${ }^{*} P<0.05,{ }^{* *} P<0.01,{ }^{* *} P<0.001,1$-way ANOVA, Tukey's correction. In box-and-whisker plots, the lower and upper margins of each box define the 25th and 75th percentiles, respectively; the internal line defines the median, and the whiskers define the range. Values outside 1.5 times the interquartile distance are shown.

natriuretic peptide (Nppa), brain natriuretic peptide ( $N p p b)$, and $\beta$-myosin heavy chain $(M y h 7)(26,34)$, was significantly increased in Fbn1 ${ }^{\mathrm{Cl} 1039 G /+}$ hearts when compared with WT hearts subjected to TAC (Supplemental Figure 3D) but was comparable between sham groups. We found that though cardiac expression of Fbn1 mRNA was significantly increased in Fbn $1^{\mathrm{Cl} 1039 \mathrm{G} /+}$ mice and WT controls in response to TAC (Figure 2A), deposition of fibrillin-1 protein in the interstitial pericapillary and perivascular spaces adjacent to cardiac 
A $\square$ Nuclei $\square$ Myocyte

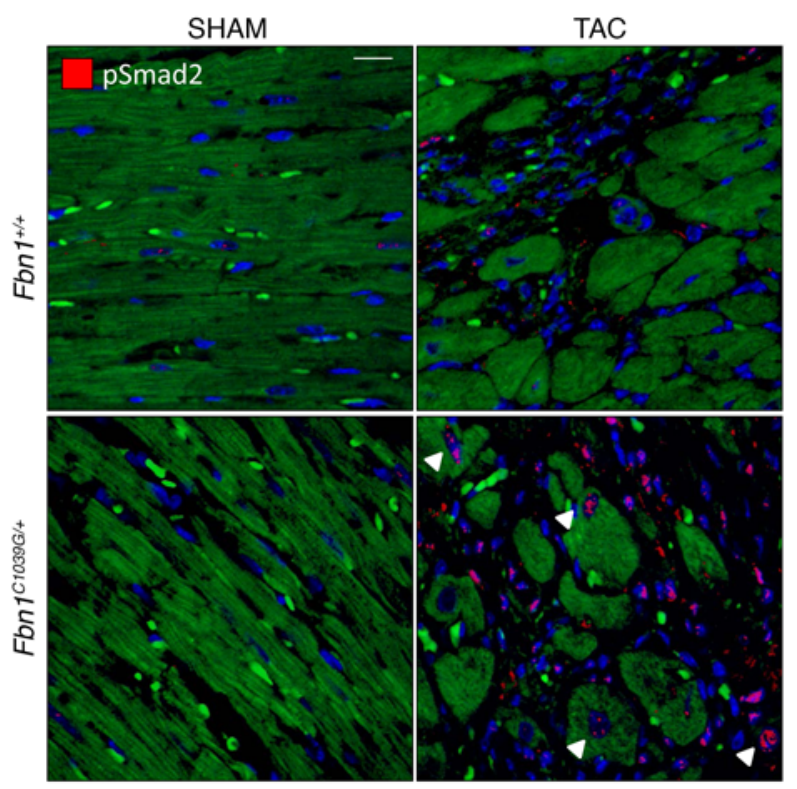

C

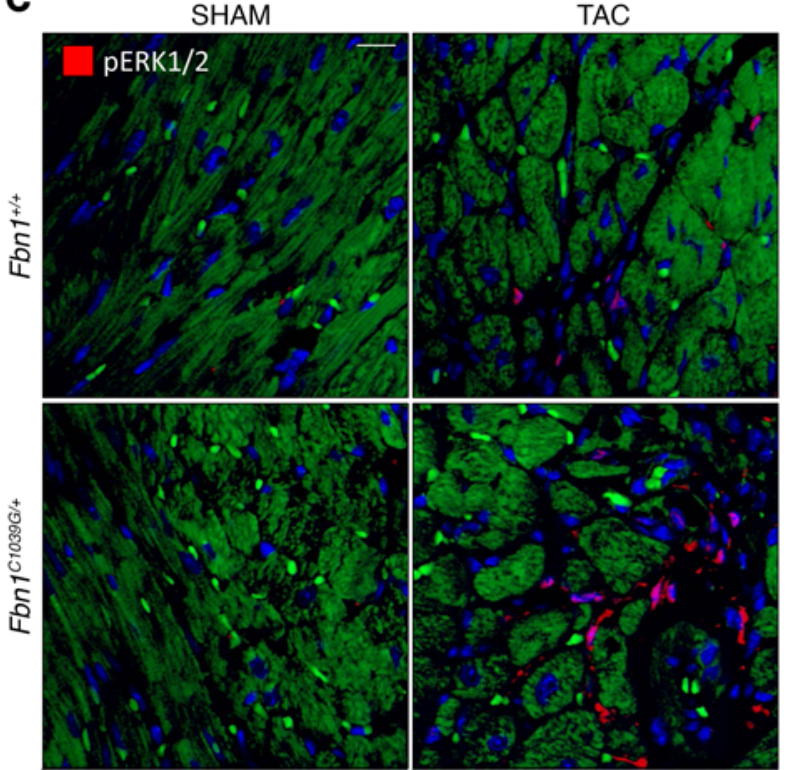

B
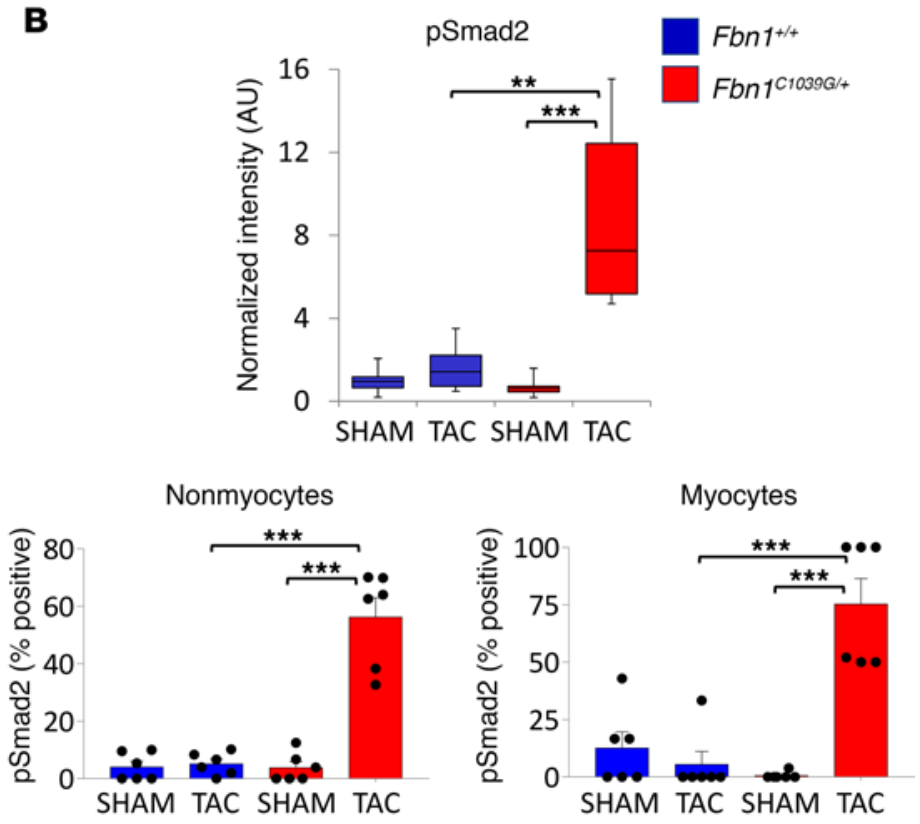

D
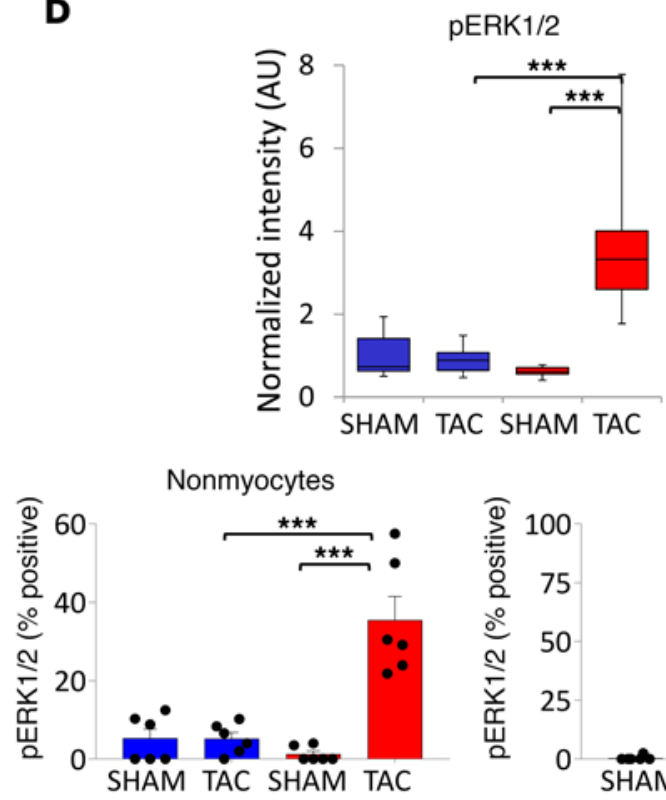

Figure 3. Smad2 and ERK1/2 activation are differentially increased in the myocyte and nonmyocyte compartments of failing Fbn ${ }^{110396 /+}$ hearts. (A) Representative sections of pSmad2 (red) immunostaining. Scale bar: $20 \mu \mathrm{m}$. White arrowheads point to examples of positively stained myocyte nuclei. (B) Summary quantification of total intensity normalized to $\mathrm{Fbn}^{++/}$:sham and labeling index (percentage positive cells) of pSmad2 in the nonmyocyte and myocyte compartments. (C and D) Representative sections and summary quantification of pERK1/2 (red) immunostaining. Scale bar: $20 \mu \mathrm{m}$. $n=6$ fields per group. ${ }^{* *} P<0.01,{ }^{* *} P<0.001,1$-way ANOVA, Tukey's correction.

nonmyocytes was increased in the compensated WT heart but not in decompensated Fbn $1^{\text {C1039G/+ }}$ hearts exposed to TAC (Figure 2, B and C, and Supplemental Figure 4). Thus, while MFS hearts have no demonstrable cardiac dysfunction at baseline, they do display an abnormal response to mechanical load, revealing that fibrillin-1 plays a critical role in the adaptive response to hemodynamic stress.

Canonical TGF- $\beta$ signaling contributes to load-induced cardiac decompensation in Fbn $1^{\mathrm{Cl039G/+}}$ mice. To gain insight into the mechanism of load-induced heart failure in $\mathrm{Fbn}^{\mathrm{C} 1039 \mathrm{G} /+}$ mice, we measured changes in the activation of the Smad2 and ERK1/2 signaling pathways, which are known to mediate the progression of aortic aneurysm in MFS mice $(12,16)$. Immunoblots showed increased levels of pSmad2 and pERK1/2 in Fbn1 $1^{C 1039 G /+}$ 
A
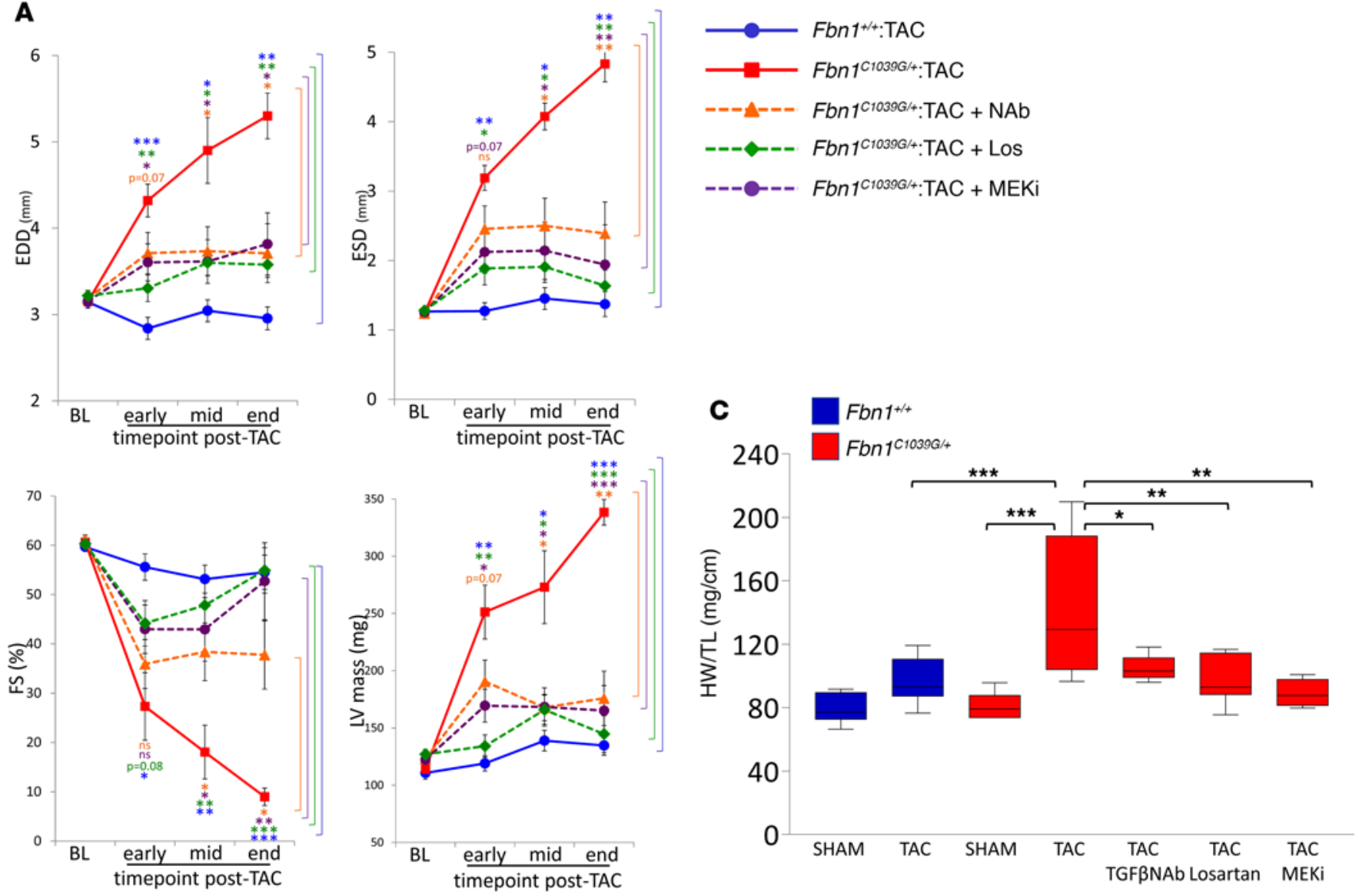

B

SHAM

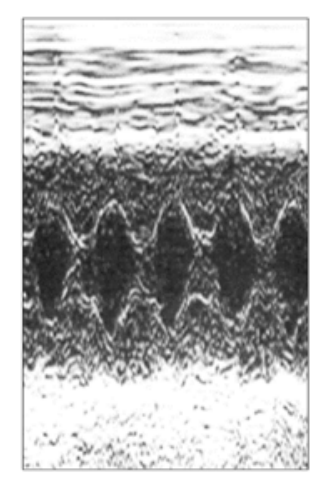

TAC

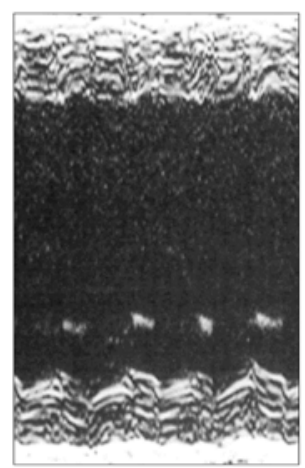

+ TGF $\beta N A b$

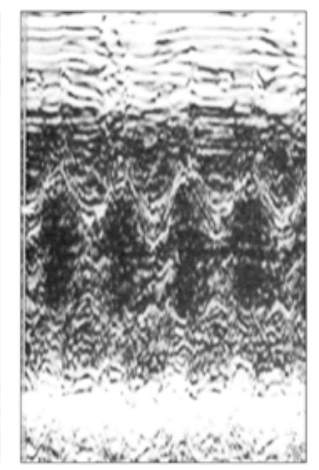

+ Losartan

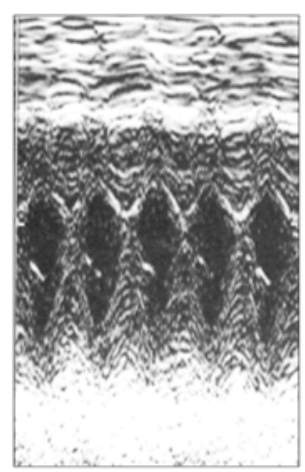

+ MEKi

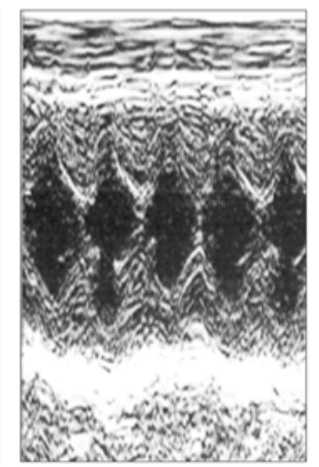

Figure 4. Treatment with TGF- $\beta$ NAb, losartan, or MEKi improves load-induced cardiac decompensation in Fbn $1{ }^{10396 /+}$ hearts, despite persistent load. (A) Temporal changes of cardiac dimensions and function of $F b n 7^{10396 /+}$ hearts after TAC, with treatment arms. Fbn ${ }^{+/+}$, blue circle with solid line; Fbn $7^{(10396 /+}$, red square with solid line; Fbn $7^{710396 /+}$ with TGF- $\beta$ NAb treatment, orange triangle with dotted line; Fbn $7^{10396 /+}$ with losartan (Los) treatment, green diamond with dotted line; Fbn $7^{(10396 /+}$ with MEK1/2 inhibitor (MEKi) treatment, purple circle with dotted line. Early time point, 1 week after TAC; mid time point, 2-4 weeks after TAC; end time point, 3-5 weeks after TAC. EDD, end-diastolic diameter; ESD, end-systolic diameter; FS, fractional shortening; LV mass, left ventricular mass; BL, baseline. Comparison pairs shown in brackets on right side of respective panel. $n \geq 5$ per group. (B) Representative M-mode echocardiograms at end time point, Fbn $7^{C 10396 /+}$ sham vs. TAC groups, with and without treatment. (C) Summary quantification of heart weight normalized to tibia length (HW/TL). $n \geq 5$ per group. ${ }^{*} P<0.05,{ }^{* *} P<0.01,{ }^{* *} P<0.001,1$-way ANOVA, Tukey's correction.

hearts when compared with WT controls after 4 weeks of TAC (Figure 2, D and E). Increased expression of prototypical TGF- $\beta$-driven gene products, such as Colla1, Postn, Serpin1, Ctgf, Spp1, and Fn1 (Figure 2F), was also observed in $F b n 1^{C 1039 G /+}$ :TAC hearts when compared with WT:TAC controls. In situ analyses of heart sections by immunofluorescence showed increased pSmad2 and pERK1/2 in Fbn $1^{\mathrm{C} 1039 \mathrm{~S}^{\prime /}}$ :TAC hearts when compared with WT:TAC controls (Figure 3). Furthermore, in Fbn $1^{1039 G / 4}$ :TAC hearts, pSmad2 colocalized 
A
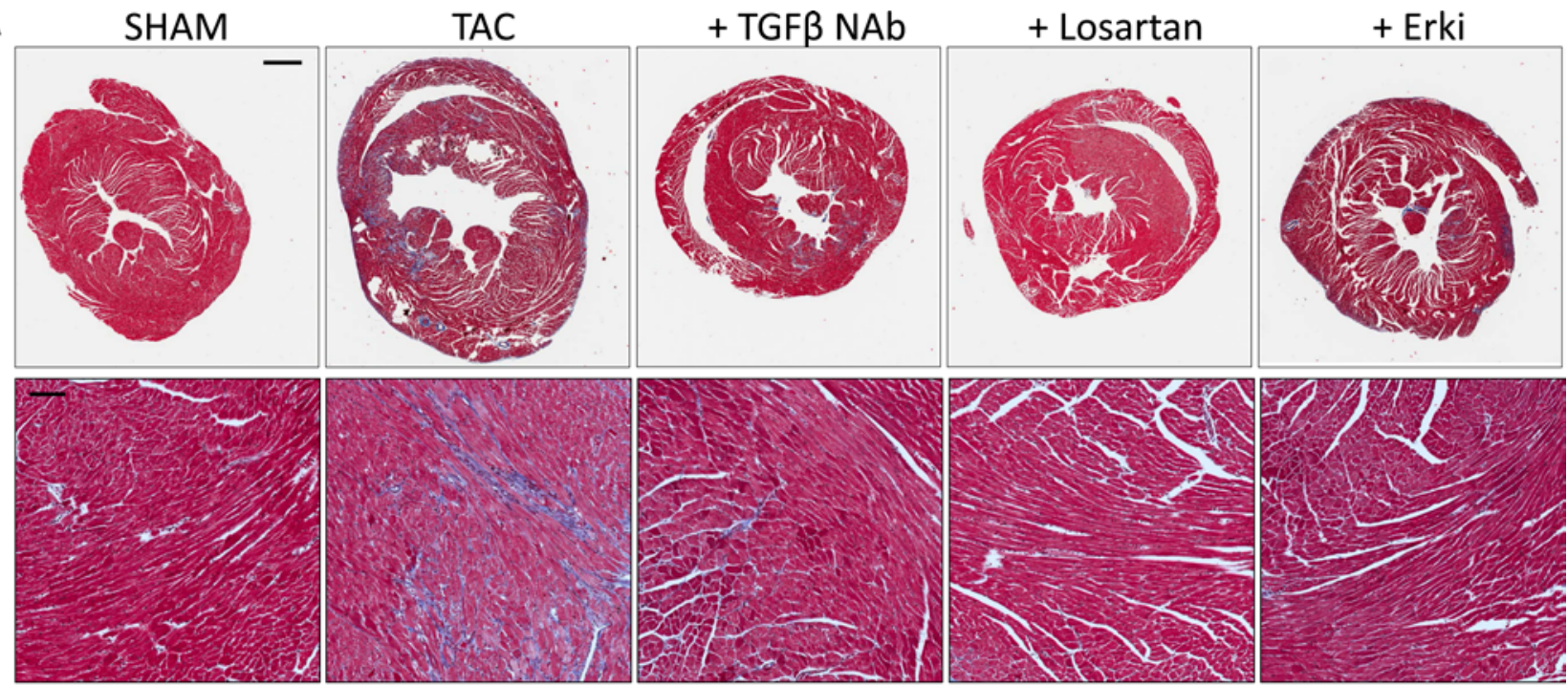

B
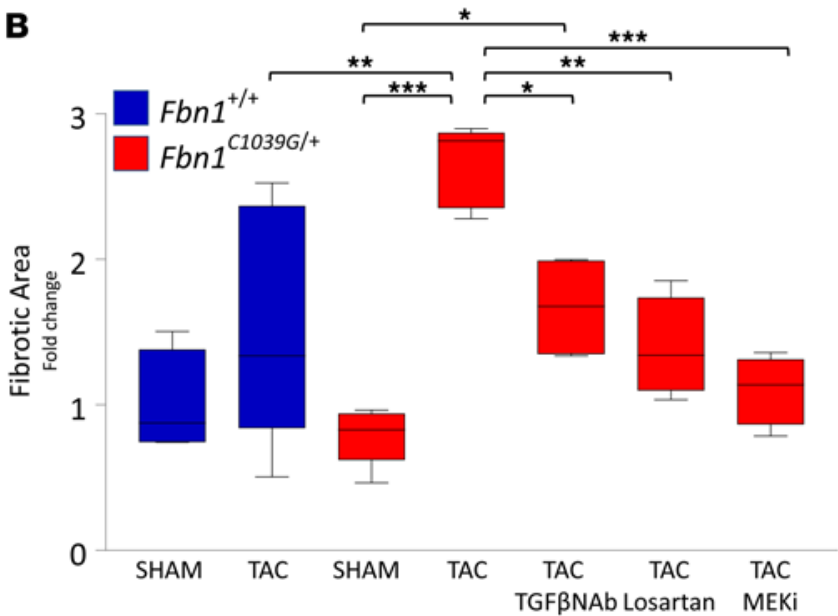

\section{15}
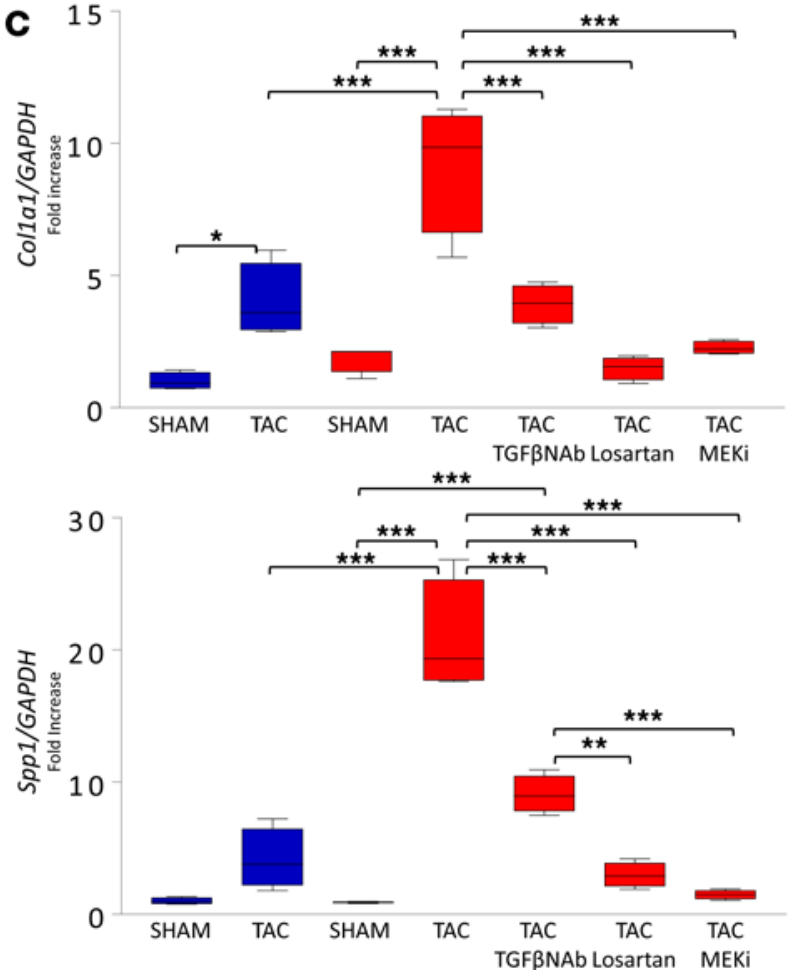

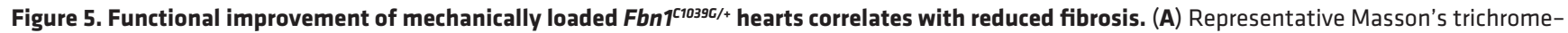
stained heart sections of $F b n 7^{C 1039 C /+}$ sham vs. TAC groups, with and without treatment, showing fibrosis stained in blue. Scale bars: $750 \mu \mathrm{m}$ (top); $100 \mu \mathrm{m}$

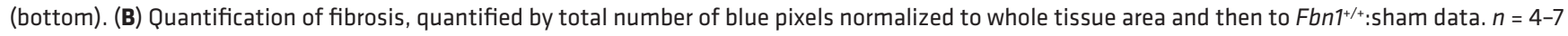
per group. (C) mRNA expression of TCF- $\beta$-responsive fibrogenic genes, normalized to Gapdh and then to Fbn1 ${ }^{+++}$: sham data, assessed by real-time RT-PCR. Col1a1, collagen type 1 a1; Spp1, osteopontin. $n=4-6$ per group. ${ }^{*} P<0.05,{ }^{* *} P<0.01,{ }^{* * *} P<0.001$, 1-way ANOVA, Tukey's correction. In box-and-whisker plots, the lower and upper margins of each box define the 25th and 75th percentiles, respectively; the internal line defines the median, and the whiskers define the range. Values outside 1.5 times the interquartile distance are shown.

with both nonmyocyte and myocyte compartments (Figure 3, A and B; Supplemental Figure 5, A and B, top; and Supplemental Figure 5, C and D, left), whereas enhanced pERK1/2 colocalized predominantly with the nonmyocyte compartment (Figure 3, C and D; Supplemental Figure 5, A and B, bottom; and Supplemental Figure 5, C and D, right). Further characterization of pSmad2-positive and pERK1/2-positive nonmyocytes showed positive costaining of vimentin in a subset of cells (Supplemental Figure 5D), suggesting nonmyocytes to be of fibroblast, endothelial, or myeloid origin (35). 
We next assessed for contributions of TGF- $\beta$, AT1R, and ERK1/2 signaling by pretreating Fbn ${ }^{C 1039 G /+}$ mice systemically with a pan-specific TGF- $\beta$-neutralizing polyclonal antibody (NAb) (12), an ARB (losar$\tan )(12)$, or a selective MEK1/2 inhibitor (MEKi, PD98059 or RDEA119) (16, 36), respectively. Cultured cardiac fibroblasts were used to show that MEKi antagonizes phosphorylation of ERK1/2 but not Smad2 (Supplemental Figure 6) as expected (16, 37, 38).

Serial echocardiography showed that NAb, losartan, or MEKi treatment substantially improved end-diastolic and end-systolic dimensions, fractional shortening, and left ventricular mass in Fbn1 ${ }^{C 1039 G /+}$ :TAC hearts when compared with vehicle-treated counterparts (Figure 4). Therapeutic effects correlated with a significant reduction in fibrotic area (Figure 5, A and B). Furthermore, TAC-induced upregulation of TGF- $\beta$-responsive fibrogenic genes, collagen (Colla1) and osteopontin (Spp1) (39-41), was attenuated by $\mathrm{NAb}$ treatment and abrogated by either losartan or MEKi (Figure 5C). Curiously, myocyte size, which

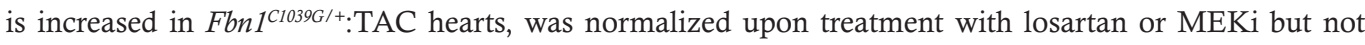
with NAb (Supplemental Figure 7, A-C). Paralleling these observations, the hypertrophic gene program induced by TAC in $\mathrm{Fbn}^{\mathrm{C1039G/+}}$ mice was unaltered by NAb treatment but was significantly suppressed by either losartan or MEKi (Supplemental Figure 7D). Thus, the comparable therapeutic efficacy of NAb, losartan, and MEKi associate with a reduction of fibrosis but not with myocyte hypertrophy.

We next examined Smad2 and ERK1/2 signaling in Fbn $1^{C 1039 G /+}$ hearts subjected to TAC and treated with NAb, losartan, or MEKi in an attempt to define the pathogenic sequence that culminates in cardiac decompensation. The therapeutic efficacies of NAb, losartan, and MEKi correlated with reduced levels of pSmad2 (Figure 6, A-D), supporting the notion that proximal events in TGF- $\beta$ signaling contribute to load-induced heart failure. However, with NAb treatment, $\mathrm{pERK} 1 / 2$ levels were unaltered (Figure $6, \mathrm{~A}$ and B), suggesting that ERK1/2 activation occurs independently or upstream of TGF- $\beta$ signaling. Informatively, MEKi treatment normalized both $\mathrm{pERK} 1 / 2$ and $\mathrm{pSmad} 2$ levels, suggesting that ERK1/2 signaling contributes directly or indirectly to induction of TGF- $\beta$ canonical signaling. Losartan also normalized both pERK1/2 and pSmad2 levels, suggesting an AT1R:ERK1/2:Smad2 axis of activation (Figure 6, C-E). In further support of an AT1R:ERK1/2:Smad2 axis of activation, we show that AngII treatment of murine cardiac fibroblasts after 24 hours results in increased activation of ERK1/2 but not Smad2 (Supplemental Figure 8). Whether or not these events represent cell-autonomous relationships remained to be determined.

Losartan or MEKi potently suppresses Smad2 activation in both nonmyocyte and myocyte compartments. To determine relationships of signaling pathways in nonmyocyte and myocyte compartments, we performed in situ analysis of pSmad 2 and pERK1/2 in Fbn1 $1^{C 1039 G /+}$ :TAC hearts treated with NAb, losartan, or MEKi. $\mathrm{NAb}$ treatment achieved a marked decrease in nonmyocyte Smad2 activation, whereas myocyte Smad2 activation largely persisted (Figure 7A and Supplemental Figure 9A). In contrast, treatment with both losartan and MEKi resulted in full normalization of pSmad2 in both cellular compartments. Losartan or MEKi achieved a marked decrease in nonmyocyte ERK1/2 activation; however, NAb had no effect (Figure 7B and Supplemental Figure 9B). All three treatments reduced vimentin reactivity (Figure 7) and prevented TAC-induced expansion of the nonmyocyte compartment in $F b n 1^{C 1039 G /+}$ mice (Supplemental Figure 9C). The failure of $\mathrm{NAb}$ to suppress myocyte Smad2 signaling likely reflects a bioavailability issue as previously discussed (42). Taken together, these data are consistent with a model that invokes a primary increase in AT1R-mediated ERK1/2 activation in nonmyocytes that is required for subsequent Smad2 activation in both nonmyocytes and myocytes.

ERK1/2 activation is pivotal in the progression of load-induced heart failure and upregulates TGF- $\beta$ ligand expression. We postulated that ERK1/2 signaling may induce nonmyocyte TGF- $\beta$ ligand production, thereby driving canonical TGF- $\beta$ signaling in both an autocrine and paracrine manner. In keeping with this hypothesis, we found that TAC increased expression of $\mathrm{Tg} f b 2$ and $\mathrm{Tg} f b 3$ in $F b n 1^{\mathrm{Cl039G/+}}$ hearts that was normalized with losartan or MEKi but not NAb (Figure 8). Furthermore, in situ analysis demonstrated that the expression of TGF- $\beta 3$ mRNA localized predominantly to the nonmyocyte compartment (Supplemental Figure 10, A and B), similarly to that of vimentin, a mesenchymal cell marker that is upregulated in fibroblast-tomyofibroblast transformation (43) (Supplemental Figure 10C). Cultured cardiac fibroblasts demonstrate a 12-fold higher expression of $T g f b 3$ compared with cultured cardiomyocytes (Supplemental Figure 10D). AngII treatment of cardiac fibroblasts after 12 hours resulted in ERK1/2 activation but not direct Smad2 activation, while TGF- $\beta 3$ treatment showed intact Smad 2 activation (Supplemental Figure 11). Furthermore, cardiac fibroblasts cultured from $\mathrm{Fbn}^{\mathrm{ClO} 103 \mathrm{G} / \mathrm{+}}$ hearts demonstrated even greater sensitivity to ERK1/2 activation in response to AngII treatment than WT hearts (Supplemental Figure 11). Taken together, these data identify 
A
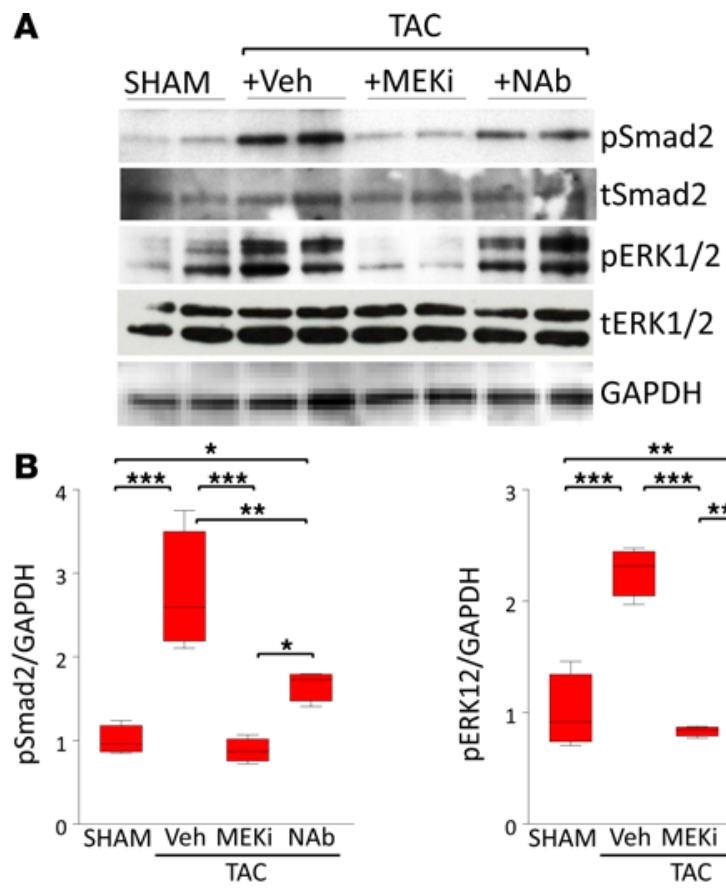

C

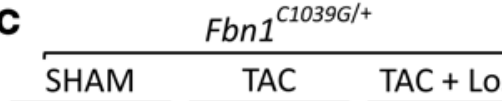

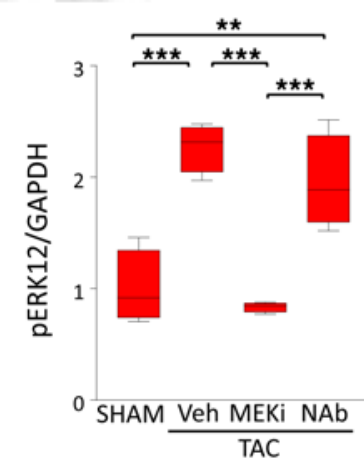

\section{SHAM $\mathrm{Fbn1}^{+/+}$}

E

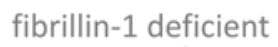

fibrillin-1 deficient matrix
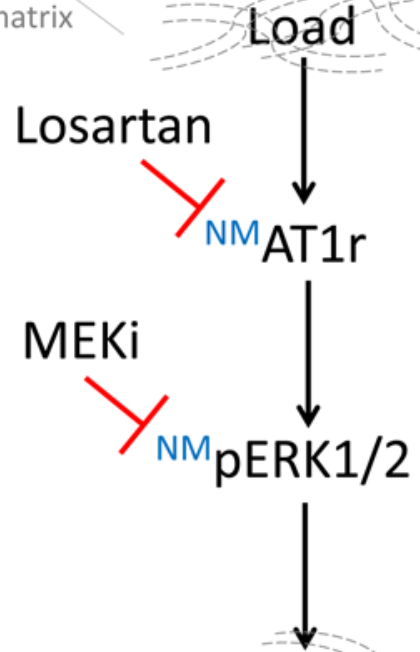

TGF $\beta$
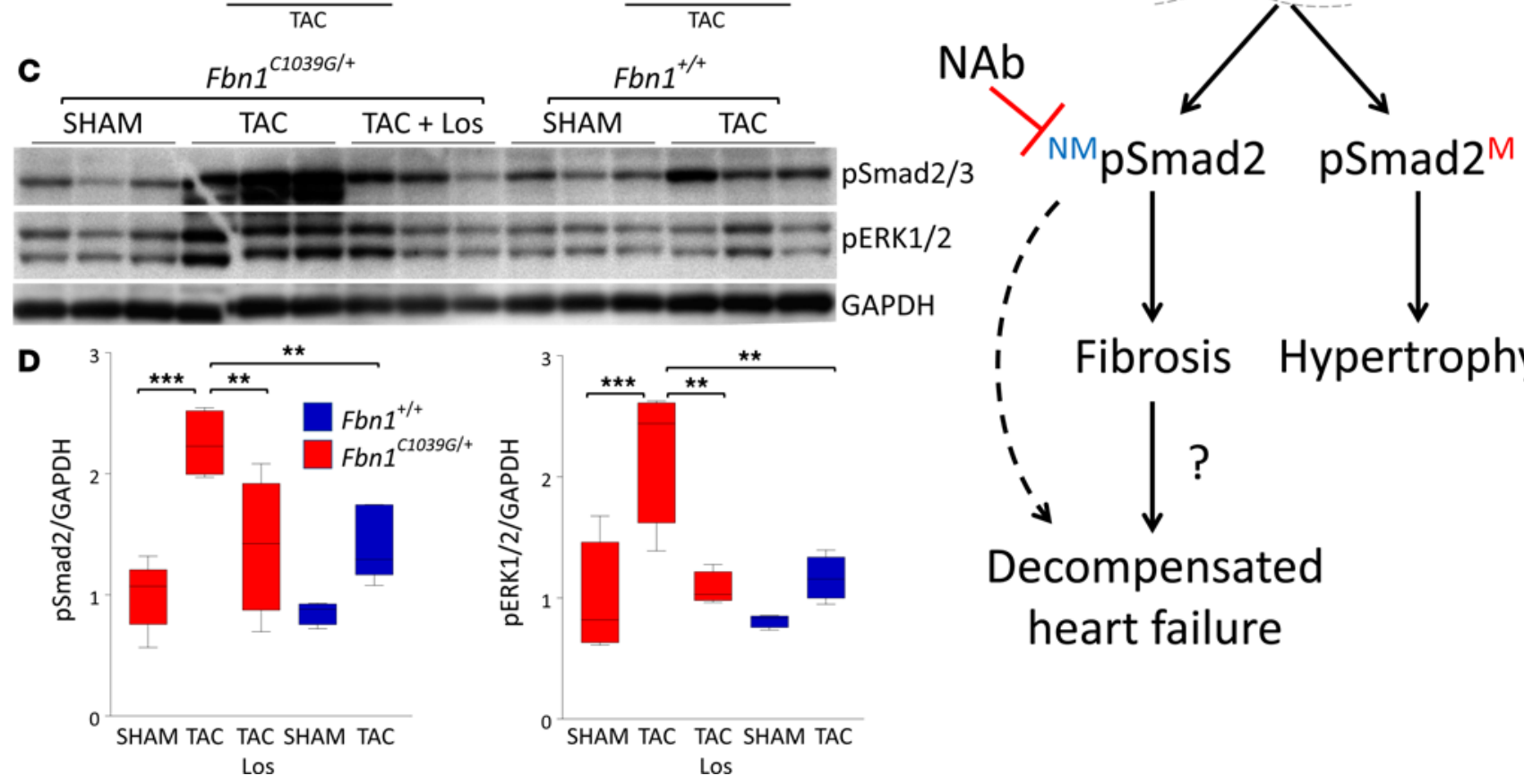

Fibrosis Hypertrophy

Figure 6. ERK1/2 activation is upstream of Smad2 activation in Fbn 1 ${ }^{\text {c10396/+ }}$ hearts. (A-D) Representative Western blot and summary quantification for Smad2 and ERK1/2 phosphorylation from left ventricular tissue lysates. $n=4-6$ per group. ${ }^{*} P<0.05,{ }^{* *} P<0.01$, ${ }^{* *} P<0.001,1$-way ANOVA, Tukey's

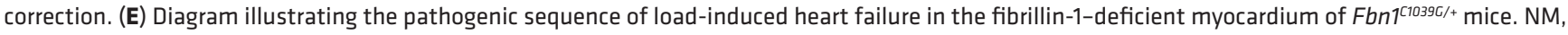
nonmyocytes; M, myocytes. In box-and-whisker plots, the lower and upper margins of each box define the 25th and 75th percentiles, respectively; the internal line defines the median, and the whiskers define the range. Values outside 1.5 times the interquartile distance are shown.

a mechanism by which ERK1/2 activation may promote the deleterious effects of TGF- $\beta$ signaling. This may be particularly important in the fibrillin-1-deficient myocardium of the hemodynamically loaded MFS heart.

\section{Discussion}

While prior reports have suggested a tendency for cardiac decompensation in MFS, it has been difficult to determine with confidence if this relates to an intrinsic myocardial predisposition, the influence of valve dysfunction, or a combination of the two. This ambiguity relates to the high frequency of mitral 
A Nuclei Myocyte

SHAM TAC + TGF $\beta N A b$ + Losartan + MEKi
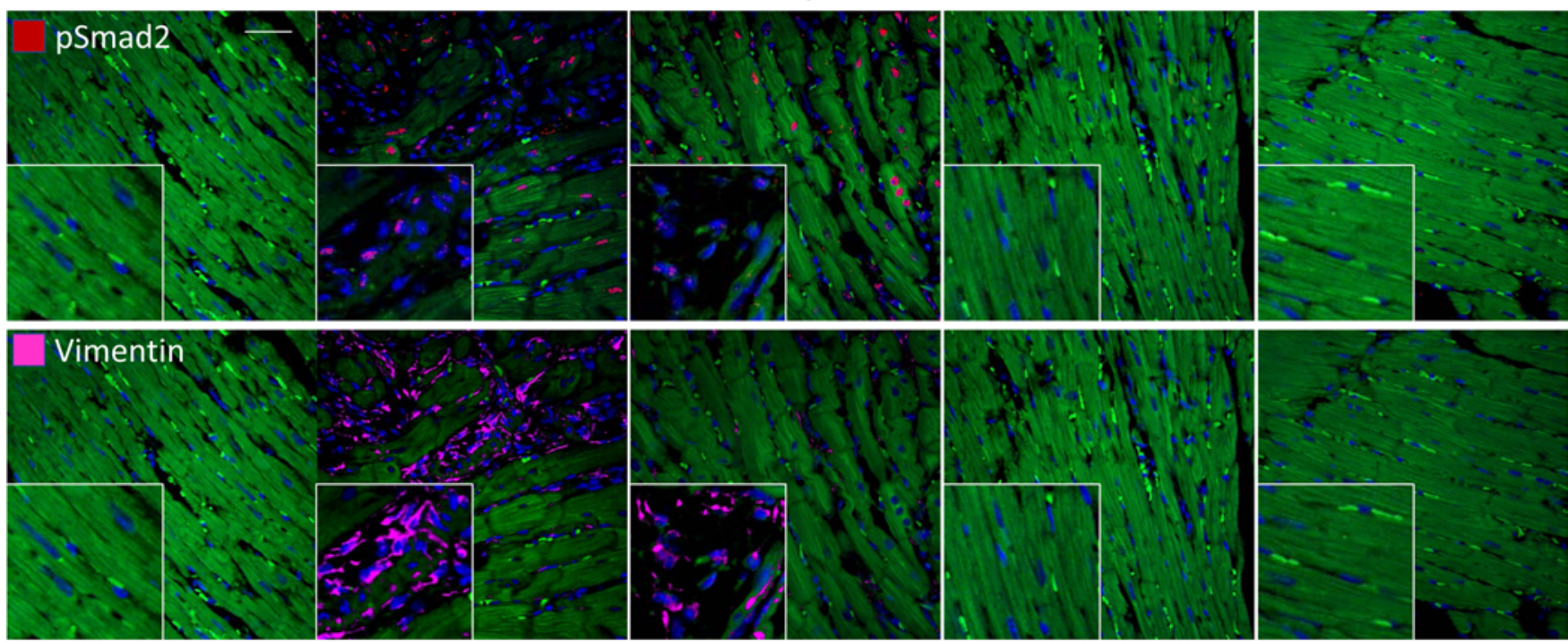

$\mathbf{B}$

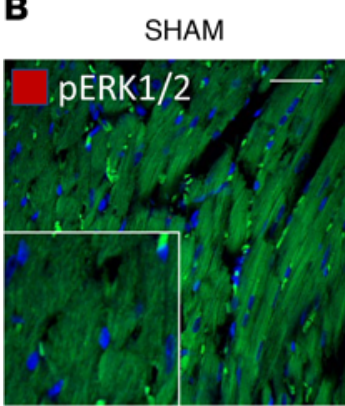

TAC

+ TGF $\beta N A b$

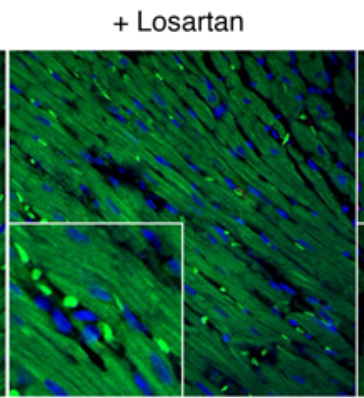

+ MEKi
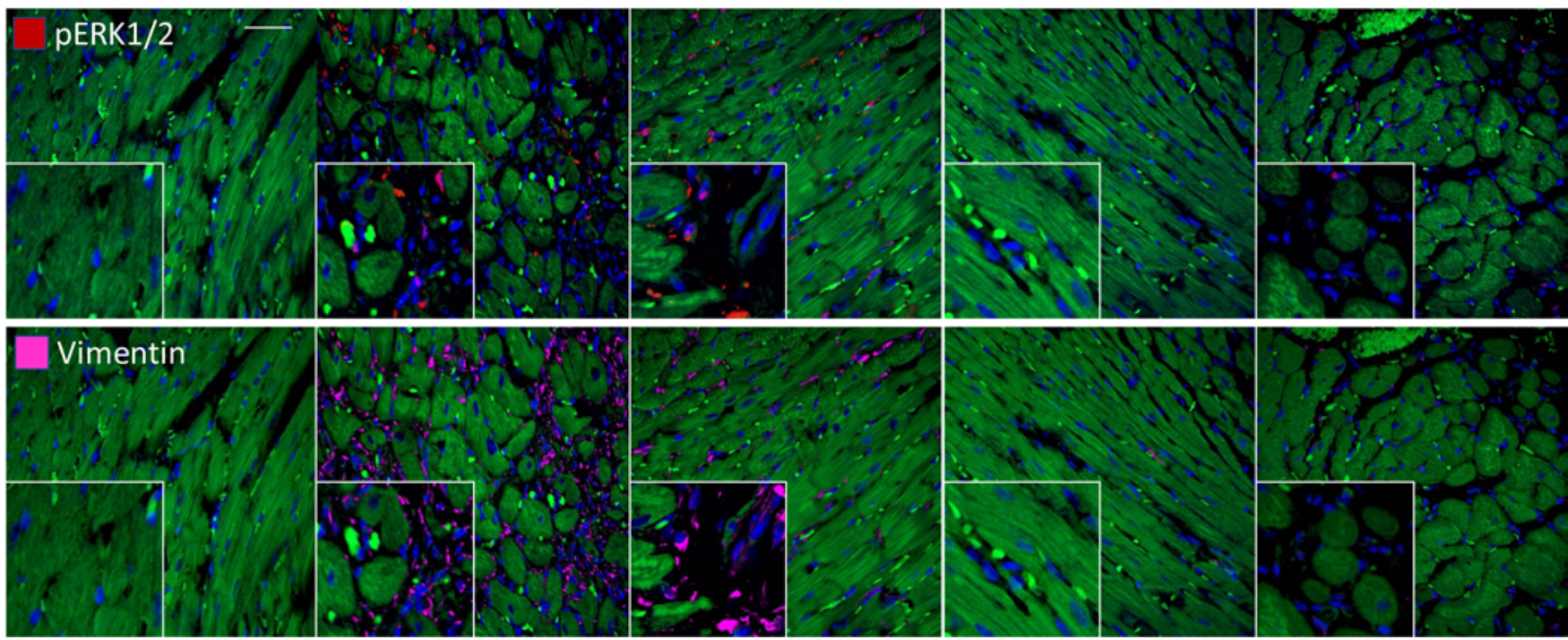

Figure 7. Inhibition of ERK1/2 activation suppresses Smad2 activation in both nonmyocyte and myocyte compartments. Representative coimmunostaining of (A) pSmad2 (red, row 1) and vimentin (pink, row 2) and of (B) pERK1/2 (red, row 1) and vimentin (pink, row 2) of myocyte-enriched sections of $\mathrm{Fbn} 7^{\mathrm{C1039C/+}}$ hearts subjected to sham vs. TAC, with treatment groups. Scale bars: $20 \mu \mathrm{m}$; zoom inset: $\times 2$ of nonmyocyte-enriched areas. Blue, DAPI (nuclei); green, lipofuscin (myocytes).

and/or aortic valve regurgitation in people and mice with MFS that can be difficult to quantitate and the use of mouse models with targeted disruption of both $F b n 1$ alleles that show accelerated cardiovascular disease (32) but uncertain mechanistic relevance to Marfan patients with heterozygous mutations and a deficiency of extracellular fibrillin-1 that progresses postnatally. In keeping with a critical contribution of hemodynamic stress, heart failure and infantile death in children with the most severe presentation of MFS uniformly associate with significant volume overload $(44,45)$ and can be delayed or even prevented by aggressive surgical intervention for valve dysfunction (46-48). In this study, we utilized a standardized PO (via TAC) as a conditional provocation in young mice heterozygous for a typical MFS-associated missense mutation in fibrillin-1. Importantly, these animals had no abnormality of heart structure or function at the onset of the study, and only mice that did not have any valvular regurgitation during 


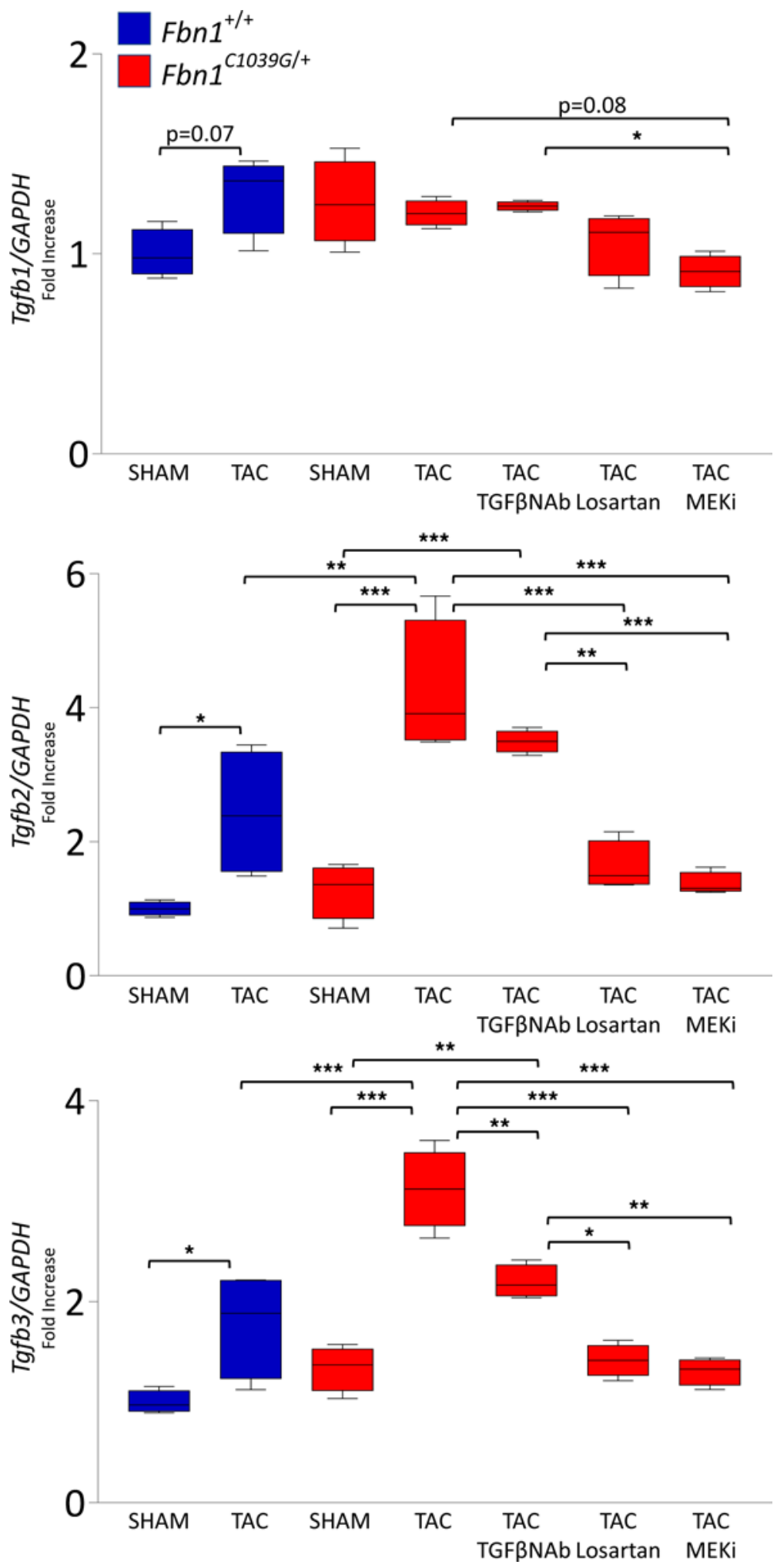

Figure 8. Inhibition of ERK1/2 activation suppresses expression of all three TGF- $\beta$ isoforms. mRNA expression of Tgfb1 (TCF- $\beta 1$ ), Tgfb2 (TCF- $\beta 2$ ), and Tgfb3 (TGF- $\beta 3$ ) isoforms, normalized to Gapdh and then to $F b n 1^{+/+}$: sham data, assessed by real-time RT-PCR. $n=4-6$ per group. ${ }^{*} P<0.05,{ }^{* *} P<0.01,{ }^{* * *} P$ $<0.001$, 1-way ANOVA, Tukey's correction. In box-and-whisker plots, the lower and upper margins of each box define the 25th and 75 th percentiles, respectively; the internal line defines the median, and the whiskers define the range. Values outside 1.5 times the interquartile distance are shown.

the entire follow-up period were analyzed. MFS mice exposed to TAC showed acute and profound DCM and heart failure, while their WT counterparts remained fully compensated. Furthermore, decompensation in MFS associated with aberrant activation of cellular signaling in the myocardium, including both the TGF- $\beta$ and ERK1/2 pathways. Pharmacologic attenuation of aberrant signaling with TGF- $\beta$, AT1R, or ERK1/2 antagonists preserved heart structure and function, despite a fixed $\mathrm{PO}$, suggesting that predisposition does not simply relate to a chronic deficiency in the structure and hence mechanical integrity of the ECM, but rather reflects dynamic but modifiable compensatory cellular signaling events that attend myocardial remodeling. These data suggest a number of conclusions with strong potential for clinical relevance. First, the concept that the myocardium is sensitized to acute hemodynamic stress in MFS supports consideration of more frequent cardiovascular surveillance for individuals with volume overload and aggressive avoidance of hypertension for indications beyond suppression of aneurysm progression. Second, the observation that mild cardiovascular stress selectively tips the MFS heart into profound failure supports the earlier application of methods to more accurately quantify valvular dysfunction (such as cardiac MRI) in patients with MFS. The thresholds used to determine when valve repair or replacement should be performed should be reconsidered in this context. Finally, as discussed below, a refined understanding of the molecular pathogenesis of heart failure in MFS may allow the testing and implementation of novel medical therapies for this and related disorders.

Prior work performed using a mouse model that is homozygous for a hypomorphic Fbn1 allele that expresses normal but reduced levels of $F b n 1$, diminishing the total amount of produced fibrillin to about $15 \%$ of normal from the time of conception, attributed the relatively mild cardiac dysfunction that was observed to altered mechanosensing and ultimately enhanced ERK1/2 signaling by cardiomyocytes (32). The focus on this cell type stemmed from the observation of DCM in a mouse line in which both copies of a conditional (flanked by loxP) Fbn1 allele were deleted through expression of Cre-recombinase using a cardiac myosin heavy chain promoter element ( $\alpha$ MHC-Cre). These experiments were difficult to interpret with certainty; however, the use of this Cre driver was also associated with a substantial $(\sim 50 \%)$ reduction in fibrillin-1 expression in cardiac fibroblasts that was equivalent to that observed in mice 
with germline haploinsufficiency for Fbn1 (32). Furthermore, isolated expression of Cre-recombinase in the myocardium has previously been associated with predisposition for cardiomyopathy $(49,50)$. While control experiments by Cook and colleagues suggested that neither isolated Fbn1 haploinsufficiency nor Cre-recombinase expression was sufficient to induce cardiomyopathy in their hands, the potential pathogenic influence of these events in combination was not tested (32). Our study now documents overt pathogenic significance for ERK1/2 signaling in load-induced heart failure in Marfan mice, given that specific antagonism with a MEKi prevented all perturbations of myocardial architecture and function. A number of observations, however, focus attention on the nonmyocyte compartment. First, application of PO in WT animals was associated with pronounced upregulation of fibrillin-1 expression, with increased deposition in the nonmyocyte compartment; failure of this event in MFS mice correlated with adverse cardiac remodeling and failure. Second, our application of in situ-based methods revealed that excessive ERK1/2 activation is specific to nonmyocytes in the failing hearts of MFS mice. Finally, the attenuation of disease seen upon treatment with TGF- $\beta$ NAb was associated with inhibition of canonical Smad signaling in nonmyocytes but not myocytes, presumably due to limited bioavailability in the latter, as has previously been reported (42).

The pathogenic sequence for load-induced heart failure in nonmyocytes of MFS mice appears to initiate with increased ERK1/2 activation through AT1R signaling. In theory, this could relate to increased expression of factors along the angiotensin-converting enzyme/AngII/AT1R axis in response to the combined influence of an altered matricellular environment and mechanical stress. What is both clear and surprising is that ERK1/2 signaling drives TGF- $\beta$ ligand production (largely TGF-2 and TGF- $\beta 3$ ) by inference in nonmyocytes and increases canonical TGF- $\beta$ signaling in both the nonmyocyte and myocyte compartments. This is in contrast to previous studies in the aorta of MFS mouse models that have shown that excessive ERK1/2 activation is, at least in part, dependent upon and therefore downstream of TGF- $\beta$ $(14,16,51)$. Of greater importance, this work shows that, similar to findings in mice with hypertrophic cardiomyopathy (52), load-induced nonmyocyte expansion, fibrosis, and heart failure in MFS mice are greatly attenuated by specific antagonism of TGF- $\beta$ using NAb; the substantial residual Smad 2 activation in myocytes but not nonmyocytes (likely on the basis of differential bioavailability of NAb) infers specific pathogenic significance in the progression of heart failure for the latter. Prevention of myocyte hypertrophy parallels the attenuation of myocyte Smad2 signaling seen with treatment with either ARB or MEKi (but not with TGF- $\beta$ NAb) and suggests that AT1R- and ERK1/2-dependent nonmyocyte secretion of TGF- $\beta$ ligands drives hypertrophy via paracrine mechanisms, while driving fibrosis and failure in an autocrine fashion. These data support a primary role for extracellular fibrillin-1 in compensating for ventricular injury and stress and help to establish the nonmyocyte microenvironment as a critical factor in the loaded MFS heart. These findings may also inform mechanisms relevant to other presentations of heart failure beyond MFS.

\section{Methods}

Mice. Fbn $1^{\mathrm{C1039G/+}}$ mice were developed as previously described (1) and bred on a pure C57BL/6 background (backcrossed for $>9$ generations).

TAC and drug treatments. PO was performed by surgical placement of suture around the transverse aorta as described previously (53). A 7-0 ligature was placed around the vessel using a 26-gauge needle to ensure consistent occlusion. This needle size was chosen to elicit a mild response, as initial studies using our standard TAC model (27-gauge needle) led to increased mortality in Fbn $1^{\mathrm{ClO} O 39 \mathrm{G} /+}$ mice. Size-, age-, and sex-matched (male) $F b n 1^{C 1039 G /+}$ mice and littermate $F b n 1^{+/+}$mice were randomized to receive TAC versus sham surgery (same procedure but without constriction).

For drug treatment studies, size-, age-, and sex-matched (male) $\mathrm{Fbn1}^{\mathrm{C1039G/+}}$ mice were randomized to receive drug treatment versus vehicle as follows: rabbit polyclonal TGF- $\beta$ NAb (pan-specific against TGF- $\beta 1$, TGF- $\beta 2$, and TGF- $\beta 3$ ) and control IgG (R\&D Systems) were reconstituted in PBS and administered via i.p. injection at a dose of $10 \mathrm{mg} / \mathrm{kg}$ every 10 days. The first dose was given 2 days prior to TAC. The MEKi, PD98059 (Calbiochem), was reconstituted in 25\% DMSO/50\% ethanol in PBS and administered daily i.p. at a dose of $3 \mathrm{mg} / \mathrm{kg}$, or placebo (DMSO) by was administered by i.p. injection, 5 days a week (36). The first dose was given 1 day prior to TAC. RDEA119 (NIH/National Human Genome Research Institute, Selleck Chemicals) was reconstituted in 10\% 2-hydroxypropyl- $\beta$-cyclodextrin (Sigma-Aldrich) in PBS and administered twice daily by oral gavage at a dose of $25 \mathrm{mg} / \mathrm{kg}$. The first dose was given 1 day prior to TAC. Losartan was administered daily in drinking water at a dose of $0.6 \mathrm{~g} / 1$ as previously described (12). Mice 
were euthanized at 3 weeks (TGF- $\beta$ NAb, PD98059, and losartan) or 5 weeks (RDEA119) for tissue analysis. The studies and analyses were performed blinded as to experimental group.

Echocardiography. All transthoracic echocardiography was performed in conscious mice. To assess the presence of valvular regurgitation, high-frame rate color Doppler imaging was performed before TAC and at the study end (Vevo 2100, 40-MHz mechanical transducer, VisualSonics). Rare mice that developed valvular regurgitation after TAC were excluded from all after TAC analyses. M-mode echocardiography followed reported methods (42).

In vivo hemodynamics. Cardiac function and arterial loading were assessed by PV analyses as described previously $(33,53)$. Briefly, mice were anesthetized, intubated, and mechanically ventilated. The LV apex was exposed between the seventh and eighth ribs, and a 1.4-Fr PV catheter (SPR 839; Millar Instruments Inc.) was advanced through the apex to lie along the longitudinal axis. Absolute volume was determined by saline calibration and aortic ultrasound, and data were assessed at steady state and during preload reduction. Data were analyzed with custom software.

Molecular analyses. Protein electrophoresis and immunoblot assays followed standard procedures, using cell lysis buffer (Cell Signaling) containing protease inhibitor and phosphatase inhibitor cocktail (Millipore). Assays were run on 10\% Bis-Tris gels (Bio-Rad), subjected to SDS-PAGE, and transferred to PVDF membranes. Membranes were probed for pSmad2 (3101, Cell Signaling), pERK1/2 (4377, Cell Signaling), GAPDH (ab9483, Abcam), tSmad2 (3103, Cell Signaling), and tERK1/2 (4695, Cell Signaling), followed by labeling with anti-rabbit/goat secondary antibody and visualizing by either enhanced chemiluminescence (SuperSignal West Femto, Life Technologies) or fluorescence detection (LI-COR Odyssey). Blots were quantified using ImageJ software (NIH) or the LI-COR Odyssey system.

Quantitative PCR using RT-PCR by amplification (Applied Biosystems) was used to assess RNA expression using standard procedures. The following TaqMan probes (Applied Biosystems) were used: Mm00514908_m1 (Fbn1), Mm00600555_m1 (Myh7), Mm01255747_g1 (Nppa), Mm00435304_g1 (Nppb), Mm99999915_g1 (Gapdh), Mm01178820_m1 (Tgfb1), Mm00436955_m1 (Tgfb2), Mm00436960_m1 (Tgfb3), Mm00801666_g1 (Col1a1), Mm01256744_m1 (Fn1), Mm00436767_m1 (Spp1), Mm00435858_m1 (Serpine1), Mm01192933_g1 (Ctgf), and Mm00450111_m1 (Postn). Relative quantification for each transcript was obtained by normalizing against housekeeping control transcript.

Histology. Myocardium was fixed with $10 \%$ formaldehyde, paraffin embedded, and sectioned into 5 - $\mu \mathrm{m}$-thick slices. Masson's trichrome staining was used to visualize fibrosis. Quantification of fibrosis content was performed in whole heart sections using the pen tool in Aperio ImageScope (Leica Biosystems) to exclude large vessels, epicardium, or pericardium and automated morphometric analysis of digital images and a previously validated modified positive pixel algorithm to quantify the amount of Trichrome (blue) stain (54) (Aperio ImageScope). Quantification of myocyte cross-sectional area, when performed in hematoxylin and eosin-stained sections, was performed with ImageJ. For wheat germ agglutinin (WGA) staining, slides were deparaffinized, rehydrated, and subjected to citrate-based heat-mediated antigen retrieval as previously described in (55). Sodium borohydride solution ( $1 \mathrm{mg} / \mathrm{ml}$ in PBS) was used for quenching. Slides were incubated with Alexa Fluor 647-preconjugated WGA (W32466, Invitrogen) overnight at $4^{\circ} \mathrm{C}$ and mounted using Prolong Gold mounting medium (P36934, Thermofisher). Image acquisition was performed on an EVOS epifluorescence microscope (Life Technologies). Quantification of myocyte cross-sectional area was performed using an automated algorithm with Image J, analyzing $1,040 \pm 70$ cells from 6-16 areas per mouse heart.

Immunohistochemistry. Slides with 5 - $\mu$ m-thick sections were deparaffinized with xylene and hydrated in a graded alcohol series. Slides were immersed in boiling antigen retrieval solution $(10 \mathrm{mM}$ sodium citrate buffer, $\mathrm{pH}$ 6.0) for 10 minutes and then set aside to cool in the same solution at room temperature for 30 minutes. Slides were incubated in freshly prepared sodium borohydride solution $(10 \mathrm{mg} / \mathrm{ml} \mathrm{PBS})$ for 20 minutes, incubated in $100 \mathrm{mM}$ glycine TBT (TBS containing $0.1 \%$ Triton-X 100) for 20 minutes, and then treated with Fc block (NB309) and Background-buster (NB306) from Innovex Biosciences according to the manufacturer's instructions. Slides were incubated in a humid chamber overnight at $4^{\circ} \mathrm{C}$ in the following primary antibodies, as applicable: anti-fibrillin-1 (1:100, ab53803, Abcam), anti-pSmad2 (1:100, 4-953, Millipore), and anti-pERK1/2 (1:100, 4370, Cell Signaling) diluted in TBT. After washing, slides were then incubated in a humid chamber with Alexa Fluor 594-conjugated donkey anti-rabbit IgG secondary antibody (1:500, R37119, Life Technologies) at room temperature for 30 minutes, washed, and then mounted in VECTASHIELD HardSet Mounting Medium with DAPI (H-1500, Vector Laboratories). 
When applicable, following staining with the secondary antibody, slides were further incubated with Alexa Fluor 647-preconjugated anti-vimentin antibody (1:100, Cell Signaling, 9856). Image acquisition was performed on a Zeiss AxioExaminer with 710NLO-Meta multiphoton confocal microscope at magnifications of $\times 20$ and $\times 40$.

High-resolution images of immunostaining (red fluorescence) were quantified using ImageJ. After background correction, fibrillin-1-positive staining was quantified by signal intensity (red) per unit area. The quantification of pSmad2 and pERK1/2 (red fluorescence) staining in myocytes and nonmyocytes was performed using ImageJ. Myocytes, known to exhibit robust lipofuscin autofluorescence and sarcomeric Z-lines in sodium borohydride-treated formaldehyde-fixed sections (56), were defined by the following characteristics: (a) cells with circular or oval-shaped nuclei (blue channel) centrally located within myocytes (green channel), (b) relatively large cell size, and (c) presence of sarcomeric Z-lines (56). Nonmyocytes were otherwise defined as all remaining cells with identifiable nuclei located peripheral to myocytes, with minimal autofluorescence, in addition to being located in the interstitial space. The number of positive cells (staining red for pSmad2 or pERK1/2) per total cells was manually counted in each compartment by two blinded observers in 10-15 areas per treatment condition.

In situ RNA hybridization. RNAscope technology (Advanced Cell Diagnostics) was used to perform in situ mRNA hybridization on formalin-fixed paraffin-embedded slides following the manufacturer's instructions. Briefly, 5- $\mu$ m-thick tissue sections were hybridized with Tgfb3 (406211) and Vim (457961) from RNAscope probes (Advanced Cell Diagnostics). A negative control probe and a positive control probe served as technical quality controls. Multiple images were acquired in Z-stack mode using a Zeiss 780 Laser Scanning Confocal Microscope. The in situ hybridization images are maximum intensity projections of acquired images.

Isolation of cardiac fibroblasts. Neonatal ventricles from 3-day-old mice or rats were separated and minced in ice-cold balanced salt solution, as described previously with minor modifications (57). To isolate cardiac cells, the tissues were incubated in a balanced salt solution containing $0.2 \%$ collagenase type 2 (Worthington Biochemical) for 6 minutes at $37^{\circ} \mathrm{C}$ with agitation. The digestion buffer was replaced 7 times, at which point the tissues were completely digested. The dispersed cells were incubated in T25 flasks for 120 minutes to plate nonmyocytes. Nonmyocytes that attached to the dishes were cultured in DMEM supplemented with $10 \%$ FBS and allowed to grow to confluence; then they were trypsinized and passaged at 1:4. This procedure yielded cell cultures that were almost exclusively fibroblasts by the first passage. Experiments were carried out after 1 additional passage.

Statistics. Data are presented as dot plots, with mean \pm SEM, or as box-and-whisker plots, with the lower and upper margins of each box defining the 25th and 75th percentiles, respectively; the internal line defining the median; and the whiskers defining the range. Values outside 1.5 times the interquartile distance are shown. Comparisons of multiple groups were performed using either 1-way or 2-way ANOVA, as appropriate, followed by Tukey's or Bonferroni's post-hoc correction (GraphPad Prism). Two-group analysis used an unpaired 2-tailed Student's $t$ test. Statistically significant differences (defined as $P \leq 0.05$ ) and trends (defined as $0.05<P<0.1$ ) between genotypes (WT vs. $\mathrm{CH}$ ) and respective treatment groups (sham vs. TAC vs. TAC plus drug treatment) are shown.

Study approval. All mice were cared for and protocols were performed under strict compliance with and with approved by the Animal Care and Use Committee of the Johns Hopkins University School of Medicine.

\section{Author contributions}

RR designed, directed, performed, and analyzed experiments. ET and GZ performed expert TAC and PV loop analysis. RR and DB acquired and analyzed all echocardiographic images. RR, EGM, RC, VN, PPR, JGB, EEG, CS, KLM, LM, NAA, and DIL performed experiments and analyzed data. NK provided tissue for analysis. DPJ, DAK, and HCD provided valuable guidance and expertise. RR and HCD wrote the paper. All authors approved the content and submission of this manuscript.

\section{Acknowledgments}

We thank the Johns Hopkins Institute Biomedical Sciences Core Microscope Facility, funded by NIH grants S10RR024550 and S10OD016374, for assistance in acquisition of immunofluorescence images. This research was supported by the Sarnoff Cardiovascular Research Foundation (to RR), the William S. Smilow Foundation for Marfan Syndrome Research (to HCD), and the Howard Hughes Medical Institute (to HCD). 
Address correspondence to: Rosanne Rouf, Division of Cardiology, Department of Medicine, Johns Hopkins University School of Medicine, 720 Rutland Avenue, Ross Building 809, Baltimore, Maryland 21224, USA. Phone: 410.502.2857; Email: rrouf1@jhmi.edu.

Or to: Harry C. Dietz, Institute of Genetic Medicine, Johns Hopkins University School of Medicine, MRB 539, 733 N. Broadway, Baltimore, Maryland 21205, USA. Phone: 410.614.0701; Email: hdietz@jhmi.edu.

RC's present address is: Department of Medicine, Sinai Hospital of Baltimore, Baltimore Maryland, USA.

PPR's present address is: Division of Cardiology, Medical University of Graz, Graz, Austria.

NK's present address is: Department of Cardiovascular Medicine, Gunma University Graduate School of Medicine, Maebashi, Japan.

1. Judge DP, et al. Evidence for a critical contribution of haploinsufficiency in the complex pathogenesis of Marfan syndrome. J Clin Invest. 2004;114(2):172-181.

2. Sisk HE, Zahka KG, Pyeritz RE. The Marfan syndrome in early childhood: analysis of 15 patients diagnosed at less than 4 years of age. Am J Cardiol. 1983;52(3):353-358.

3. Morse RP, et al. Diagnosis and management of infantile Marfan syndrome. Pediatrics. 1990;86(6):888-895.

4. Alpendurada F, et al. Evidence for Marfan cardiomyopathy. Eur J Heart Fail. 2010;12(10):1085-1091.

5. Kuwahara F, et al. Transforming growth factor-beta function blocking prevents myocardial fibrosis and diastolic dysfunction in pressure-overloaded rats. Circulation. 2002;106(1):130-135.

6. Meijboom LJ, et al. Evaluation of left ventricular dimensions and function in Marfan's syndrome without significant valvular regurgitation. Am J Cardiol. 2005;95(6):795-797.

7. de Witte P, et al. Intrinsic biventricular dysfunction in Marfan syndrome. Heart. 2011;97(24):2063-2068.

8. Uretsky S, et al. Quantification of left ventricular remodeling in response to isolated aortic or mitral regurgitation. J Cardiovasc Magn Reson. 2010;12:32.

9. Sakai LY, Keene DR, Engvall E. Fibrillin, a new 350-kD glycoprotein, is a component of extracellular microfibrils. J Cell Biol. 1986;103(6 Pt 1):2499-2509.

10. Neptune ER, et al. Dysregulation of TGF-beta activation contributes to pathogenesis in Marfan syndrome. Nat Genet. 2003;33(3):407-411.

11. Ng CM, et al. TGF-beta-dependent pathogenesis of mitral valve prolapse in a mouse model of Marfan syndrome. J Clin Invest. 2004;114(11):1586-1592.

12. Habashi JP, et al. Losartan, an AT1 antagonist, prevents aortic aneurysm in a mouse model of Marfan syndrome. Science. 2006;312(5770):117-121.

13. Cohn RD, et al. Angiotensin II type 1 receptor blockade attenuates TGF-beta-induced failure of muscle regeneration in multiple myopathic states. Nat Med. 2007;13(2):204-210.

14. Cook JR, et al. Dimorphic effects of transforming growth factor- $\beta$ signaling during aortic aneurysm progression in mice suggest a combinatorial therapy for Marfan syndrome. Arterioscler Thromb Vasc Biol. 2015;35(4):911-917.

15. Eguchi S, Dempsey PJ, Frank GD, Motley ED, Inagami T. Activation of MAPKs by angiotensin II in vascular smooth muscle cells. Metalloprotease-dependent EGF receptor activation is required for activation of ERK and p38 MAPK but not for JNK. J Biol Chem. 2001;276(11):7957-7962.

16. Holm TM, et al. Noncanonical TGF $\beta$ signaling contributes to aortic aneurysm progression in Marfan syndrome mice. Science. 2011;332(6027):358-361.

17. Khan S, Joyce J, Margulies KB, Tsuda T. Enhanced bioactive myocardial transforming growth factor- $\beta$ in advanced human heart failure. Circ J. 2014;78(11):2711-2718.

18. Malebranche L, et al. Enhanced bioactive tgf- levels in the myocardium suggests early pathological transition in asymptomatic severe mitral regurgitation. J Am Coll Cardiol. 2014;63(12):A922.

19. Fielitz J, et al. Activation of the cardiac renin-angiotensin system and increased myocardial collagen expression in human aortic valve disease. J Am Coll Cardiol. 2001;37(5):1443-1449.

20. Bouzeghrane F, Reinhardt DP, Reudelhuber TL, Thibault G. Enhanced expression of fibrillin-1, a constituent of the myocardial extracellular matrix in fibrosis. Am J Physiol Heart Circ Physiol. 2005;289(3):H982-H991.

21. Marijianowski MM, Teeling P, Mann J, Becker AE. Dilated cardiomyopathy is associated with an increase in the type I/type III collagen ratio: a quantitative assessment. J Am Coll Cardiol. 1995;25(6):1263-1272.

22. Brooks A, Schinde V, Bateman AC, Gallagher PJ. Interstitial fibrosis in the dilated non-ischaemic myocardium. Heart. 2003;89(10):1255-1256.

23. Rosenkranz S. TGF-beta1 and angiotensin networking in cardiac remodeling. Cardiovasc Res. 2004;63(3):423-432.

24. van Wamel AJ, Ruwhof C, van der Valk-Kokshoom LE, Schrier PI, van der Laarse A. The role of angiotensin II, endothelin-1 and transforming growth factor-beta as autocrine/paracrine mediators of stretch-induced cardiomyocyte hypertrophy. Mol Cell Biochem. 2001;218(1-2):113-124.

25. Boluyt MO, et al. Alterations in cardiac gene expression during the transition from stable hypertrophy to heart failure. Marked upregulation of genes encoding extracellular matrix components. Circ Res. 1994;75(1):23-32.

26. Parker TG, Packer SE, Schneider MD. Peptide growth factors can provoke "fetal" contractile protein gene expression in rat 
cardiac myocytes. J Clin Invest. 1990;85(2):507-514.

27. Villarreal FJ, Dillmann WH. Cardiac hypertrophy-induced changes in mRNA levels for TGF-beta 1, fibronectin, and collagen. Am J Physiol. 1992;262(6 Pt 2):H1861-H1866.

28. Shimkets RA, et al. Gene expression analysis by transcript profiling coupled to a gene database query. Nat Biotechnol. 1999;17(8):798-803.

29. Zhang H, Hu W, Ramirez F. Developmental expression of fibrillin genes suggests heterogeneity of extracellular microfibrils J Cell Biol. 1995;129(4):1165-1176.

30. Vracko R, Thorning D, Frederickson RG. Spatial arrangements of microfibrils in myocardial scars: application of antibody to fibrillin. J Mol Cell Cardiol. 1990;22(7):749-757.

31. Tae HJ, Petrashevskaya N, Marshall S, Krawczyk M, Talan M. Cardiac remodeling in the mouse model of Marfan syndrome develops into two distinctive phenotypes. Am J Physiol Heart Circ Physiol. 2016;310(2):H290-H299.

32. Cook JR, et al. Abnormal muscle mechanosignaling triggers cardiomyopathy in mice with Marfan syndrome. J Clin Invest. 2014;124(3):1329-1339.

33. Pacher P, Nagayama T, Mukhopadhyay P, Bátkai S, Kass DA. Measurement of cardiac function using pressure-volume conductance catheter technique in mice and rats. Nat Protoc. 2008;3(9):1422-1434.

34. Kuwahara K, et al. NRSF regulates the fetal cardiac gene program and maintains normal cardiac structure and function. $E M B O$ J. 2003;22(23):6310-6321.

35. Moore-Morris T, et al. Resident fibroblast lineages mediate pressure overload-induced cardiac fibrosis. J Clin Invest. 2014;124(7):2921-2934.

36. Muchir A, Shan J, Bonne G, Lehnart SE, Worman HJ. Inhibition of extracellular signal-regulated kinase signaling to prevent cardiomyopathy caused by mutation in the gene encoding A-type lamins. Hum Mol Genet. 2009;18(2):241-247.

37. Hung KY, Chen CT, Huang JW, Lee PH, Tsai TJ, Hsieh BS. Dipyridamole inhibits TGF-beta-induced collagen gene expression in human peritoneal mesothelial cells. Kidney Int. 2001;60(4):1249-1257.

38. Iverson C, et al. RDEA119/BAY 869766: a potent, selective, allosteric inhibitor of MEK1/2 for the treatment of cancer. Cancer Res. 2009;69(17):6839-6847.

39. Zahradka P. Novel role for osteopontin in cardiac fibrosis. Circ Res. 2008;102(3):270-272.

40. Ashizawa N, et al. Osteopontin is produced by rat cardiac fibroblasts and mediates A(II)-induced DNA synthesis and collagen gel contraction. J Clin Invest. 1996;98(10):2218-2227.

41. Wang D, et al. Effects of pressure overload on extracellular matrix expression in the heart of the atrial natriuretic peptide-null mouse. Hypertension. 2003;42(1):88-95.

42. Koitabashi N, et al. Pivotal role of cardiomyocyte TGF- $\beta$ signaling in the murine pathological response to sustained pressure overload. J Clin Invest. 2011;121(6):2301-2312.

43. Baum J, Duffy HS. Fibroblasts and myofibroblasts: what are we talking about? J Cardiovasc Pharmacol. 2011;57(4):376-379.

44. Booms P, et al. Novel exon skipping mutation in the fibrillin-1 gene: two 'hot spots' for the neonatal Marfan syndrome. Clin Genet. 1999;55(2):110-117.

45. Geva T, Sanders SP, Diogenes MS, Rockenmacher S, Van Praagh R. Two-dimensional and Doppler echocardiographic and pathologic characteristics of the infantile Marfan syndrome. Am J Cardiol. 1990;65(18):1230-1237.

46. Brito-Filho SL, Oporto V, Campos O, Alvares AB, Carvalho AC. A case of neonatal Marfan syndrome with good late follow-up: is it possible to avoid an early unfavourable outcome? Cardiol Young. 2013;23(2):301-303.

47. Kitahara H, Aeba R, Takaki H, Shimizu H. Palliative mitral valve repair during infancy for neonatal Marfan syndrome. Ann Thorac Surg. 2016;101(5):1987-1988.

48. Seo YJ, Lee KE, Kim GB, Kwon BS, Bae EJ, Noh CI. Infantile Marfan syndrome in a Korean tertiary referral center. Korean J Pediatr. 2016;59(2):59-64.

49. Buerger A, et al. Dilated cardiomyopathy resulting from high-level myocardial expression of Cre-recombinase. J Card Fail. 2006;12(5):392-398.

50. Davis J, Maillet M, Miano JM, Molkentin JD. Lost in transgenesis: a user's guide for genetically manipulating the mouse in cardiac research. Circ Res. 2012;111(6):761-777.

51. Habashi JP, et al. Angiotensin II type 2 receptor signaling attenuates aortic aneurysm in mice through ERK antagonism. Science. 2011;332(6027):361-365.

52. Teekakirikul P, et al. Cardiac fibrosis in mice with hypertrophic cardiomyopathy is mediated by non-myocyte proliferation and requires Tgf- $\beta$. J Clin Invest. 2010;120(10):3520-3529.

53. Takimoto E, et al. Chronic inhibition of cyclic GMP phosphodiesterase 5A prevents and reverses cardiac hypertrophy. Nat Med. 2005;11(2):214-222.

54. Krajewska M, et al. Image analysis algorithms for immunohistochemical assessment of cell death events and fibrosis in tissue sections. J Histochem Cytochem. 2009;57(7):649-663.

55. Seo K, et al. Combined TRPC3 and TRPC6 blockade by selective small-molecule or genetic deletion inhibits pathological cardiac hypertrophy. Proc Natl Acad Sci U S A. 2014;111(4):1551-1556.

56. Baschong W, Suetterlin R, Laeng RH. Control of autofluorescence of archival formaldehyde-fixed, paraffin-embedded tissue in confocal laser scanning microscopy (CLSM). J Histochem Cytochem. 2001;49(12):1565-1572.

57. Komuro I, et al. Stretching cardiac myocytes stimulates protooncogene expression. J Biol Chem. 1990;265(7):3595-3598. 